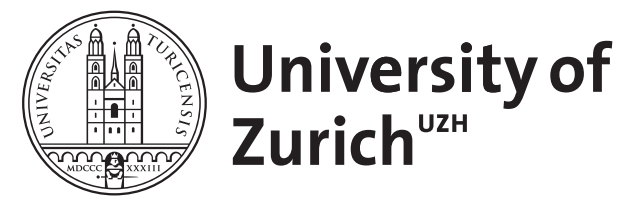

\title{
A law of large numbers approximation for Markov population processes with countably many types
}

\author{
Barbour, A D ; Luzcak, M J
}

\begin{abstract}
When modelling metapopulation dynamics, the influence of a single patch on the metapopulation depends on the number of individuals in the patch. Since the population size has no natural upper limit, this leads to systems in which there are countably infinitely many possible types of individual. Analogous considerations apply in the transmission of parasitic diseases. In this paper, we prove a law of large numbers for quite general systems of this kind, together with a rather sharp bound on the rate of convergence in an appropriately chosen weighted 1 norm.
\end{abstract}

DOI: https://doi.org/10.1007/s00440-011-0359-2

Posted at the Zurich Open Repository and Archive, University of Zurich

ZORA URL: https://doi.org/10.5167/uzh-58136

Journal Article

Accepted Version

Originally published at:

Barbour, A D; Luzcak, M J (2012). A law of large numbers approximation for Markov population processes with countably many types. Probability Theory and Related Fields, 153(3-4):727-757.

DOI: https://doi.org/10.1007/s00440-011-0359-2 


\title{
A law of large numbers approximation for Markov population processes with countably many types
}

\author{
A. D. Barbour* and M. J. Luczak ${ }^{\dagger}$ \\ Universität Zürich and London School of Economics
}

\begin{abstract}
When modelling metapopulation dynamics, the influence of a single patch on the metapopulation depends on the number of individuals in the patch. Since the population size has no natural upper limit, this leads to systems in which there are countably infinitely many possible types of individual. Analogous considerations apply in the transmission of parasitic diseases. In this paper, we prove a law of large numbers for rather general systems of this kind, together with a rather sharp bound on the rate of convergence in an appropriately chosen weighted $\ell_{1}$ norm.
\end{abstract}

Keywords: Epidemic models, metapopulation processes, countably many types, quantitative law of large numbers, Markov population processes AMS subject classification: 92D30, 60J27, 60B12

Running head: A law of large numbers approximation

\section{Introduction}

There are many biological systems that consist of entities that differ in their influence according to the number of active elements associated with them,

*Angewandte Mathematik, Universität Zürich, Winterthurertrasse 190, CH-8057 ZÜRICH; ADB was supported in part by Schweizerischer Nationalfonds Projekt Nr. 20$107935 / 1$.

${ }^{\dagger}$ London School of Economics; MJL was supported in part by a STICERD grant. 
and can be divided into types accordingly. In parasitic diseases (Barbour \& Kafetzaki 1993, Luchsinger 2001a,b, Kretzschmar 1993), the infectivity of a host depends on the number of parasites that it carries; in metapopulations, the migration pressure exerted by a patch is related to the number of its inhabitants (Arrigoni 2003); the behaviour of a cell may depend on the number of copies of a particular gene that it contains (Kimmel \& Axelrod 2002, Chapter 7); and so on. In none of these examples is there a natural upper limit to the number of associated elements, so that the natural setting for a mathematical model is one in which there are countably infinitely many possible types of individual. In addition, transition rates typically increase with the number of associated elements in the system - for instance, each parasite has an individual death rate, so that the overall death rate of parasites grows at least as fast as the number of parasites - and this leads to processes with unbounded transition rates. This paper is concerned with approximations to density dependent Markov models of this kind, when the typical population size $N$ becomes large.

In density dependent Markov population processes with only finitely many types of individual, a law of large numbers approximation, in the form of a system of ordinary differential equations, was established by Kurtz (1970), together with a diffusion approximation (Kurtz, 1971). In the infinite dimensional case, the law of large numbers was proved for some specific models (Barbour \& Kafetzaki 1993, Luchsinger 2001b, Arrigoni 2003, see also Léonard 1990), using individually tailored methods. A more general result was proved by Eibeck \& Wagner (2003). In Barbour \& Luczak (2008), the law of large numbers was strengthened by the addition of an error bound in $\ell_{1}$ that is close to optimal order in $N$. The argument makes use of an intermediate approximation involving an independent particles process, for which the law of large numbers is relatively easy to analyse. This process is then shown to be sufficiently close to the interacting process of actual interest, by means of a coupling argument. However, the generality of the results obtained is limited by the simple structure of the intermediate process, and the model of Arrigoni (2003), for instance, lies outside their scope.

In this paper, we develop an entirely different approach, which circumvents the need for an intermediate approximation, enabling a much wider class of models to be addressed. The setting is that of families of Markov population processes $X_{N}:=\left(X_{N}(t), t \geq 0\right), N \geq 1$, taking values in the countable space $\mathcal{X}_{+}:=\left\{X \in \mathbb{Z}_{+}^{\mathbb{Z}_{+}} ; \sum_{m \geq 0} X^{m}<\infty\right\}$. Each component repre- 
sents the number of individuals of a particular type, and there are countably many types possible; however, at any given time, there are only finitely many individuals in the system. The process evolves as a Markov process with state-dependent transitions

$$
X \rightarrow X+J \text { at rate } N \alpha_{J}\left(N^{-1} X\right), \quad X \in \mathcal{X}_{+}, J \in \mathcal{J},
$$

where each jump is of bounded influence, in the sense that

$$
\mathcal{J} \subset\left\{X \in \mathbb{Z}^{\mathbb{Z}_{+}} ; \sum_{m \geq 0}\left|X^{m}\right| \leq J_{*}<\infty\right\}, \quad \text { for some fixed } \quad J_{*}<\infty,
$$

so that the number of individuals affected is uniformly bounded. Density dependence is reflected in the fact that the arguments of the functions $\alpha_{J}$ are counts normalised by the 'typical size' $N$. Writing $\mathcal{R}:=R_{+}^{\mathbb{Z}_{+}}$, the functions $\alpha_{J}: \mathcal{R} \rightarrow R_{+}$are assumed to satisfy

$$
\sum_{J \in \mathcal{J}} \alpha_{J}(\xi)<\infty, \quad \xi \in \mathcal{R}_{0},
$$

where $\mathcal{R}_{0}:=\left\{\xi \in \mathcal{R}: \xi_{i}=0\right.$ for all but finitely many $\left.i\right\}$; this assumption implies that the processes $X_{N}$ are pure jump processes, at least for some non-zero length of time. To prevent the paths leaving $\mathcal{X}_{+}$, we also assume that $\alpha_{J}(\xi)=0$ if $\xi^{l}=0$ for any $l \in \mathbb{Z}_{+}$such that $J^{l} \leq-1$.

The law of large numbers is then formally expressed in terms of the system of deterministic equations

$$
\frac{d \xi}{d t}=\sum_{J \in \mathcal{J}} J \alpha_{J}(\xi)=: F_{0}(\xi)
$$

to be understood componentwise for those $\xi \in \mathcal{R}$ such that

$$
\sum_{J \in \mathcal{J}}\left|J^{l}\right| \alpha_{J}(\xi)<\infty, \quad \text { for all } l \geq 0,
$$

thus by assumption including $\mathcal{R}_{0}$. Here, the quantity $F_{0}$ represents the infinitesimal average drift of the components of the random process. It is not even immediately clear that equations (1.3) have a solution. In order to make progress, it is therefore assumed that the unbounded components in 
the transition rates can be assimilated into a linear operator $A$, in the sense that $F_{0}$ can be written in the form

$$
F_{0}(\xi)=A \xi+F(\xi)
$$

again to be understood componentwise, where $F$ is locally Lipschitz with respect to an appropriate norm. Under some further assumptions, the operator $A$ is then shown to generate a closed semigroup of transition operators. As a result of this, the deterministic system can be shown to have a mild solution, derived as a perturbation of the linear system by means of a variation of constants formula (Pazy 1983, Theorem 1.4, Chapter 6). We then show that the solution of the stochastic system can be expressed using a similar formula, but having an additional stochastic component in the perturbation. To obtain tight control over this extra element, we derive Chernoff-like bounds on the deviations of the most significant components with the help of a family of exponential martingales; the remaining components are treated using some general a priori bounds on the behaviour of the stochastic system. Then, after taking the difference between the stochastic and deterministic formulae, a Gronwall argument can be carried through, leading to the desired approximation.

The main result, Theorem 4.7, guarantees an approximation error of order $O\left(N^{-1 / 2} \sqrt{\log N}\right)$ in a weighted $\ell_{1}$ metric, except on an event of probability of order $O\left(N^{-1} \log N\right)$. More precisely, we prove that, under suitable assumptions, the following holds. For a suitable measure $\mu$ on $\mathbb{Z}^{+}$, let $\|\cdot\|_{\mu}$ be the $\ell_{1}$ norm on $\mathcal{R}$ weighted by $\mu$, and let $x$ be a mild solution to the deterministic differential equation (1.3) with respect to the norm $\|\cdot\|_{\mu}$. For each $T>0$, there exist constants $K_{T}^{(1)}, K_{T}^{(2)}, K_{T}^{(3)}$ such that for $N$ large enough, if

$$
\left\|N^{-1} X_{N}(0)-x(0)\right\|_{\mu} \leq K_{T}^{(1)} \sqrt{\frac{\log N}{N}}
$$

then

$$
\mathbf{P}\left(\sup _{0 \leq t \leq T}\left\|N^{-1} X_{N}(t)-x(t)\right\|_{\mu}>K_{T}^{(2)} \sqrt{\frac{\log N}{N}}\right) \leq K_{T}^{(3)} \frac{\log N}{N} .
$$

The error bound is sharper, by a factor of $\log N$, than in Barbour \& Luczak (2008), and the theorem is applicable to a much wider class of models. However, the method of proof involves moment arguments, which require somewhat 
stronger assumptions on the initial state of the system, and, in models such as that of Barbour \& Kafetzaki (1993), on the choice of infection distributions allowed. The conditions under which the theorem holds can be divided into three categories: growth conditions on the transition rates, so that the a priori bounds, which have the character of moment bounds, can be established; conditions on the operator $A$, sufficient to limit the growth of the semigroup, and (together with the properties of $F$ ) to determine the weights defining the metric in which the approximation is to be carried out; and conditions on the initial state of the system. The a priori bounds are derived in Section 2, the semigroup analysis is conducted in Section 3, and the approximation proper is carried out in Section 4. The paper concludes in Section 5 with some examples.

\section{A priori bounds}

We begin by imposing further conditions on the transition rates of the process $X_{N}$, sufficient to constrain its paths to bounded subsets of $\mathcal{X}_{+}$during finite time intervals, and in particular to ensure that only finitely many jumps can occur in finite time. The conditions that follow have the flavour of moment conditions on the jump distributions. Since the index $j \in \mathbb{Z}_{+}$is symbolic in nature, we start by fixing an $\nu \in \mathcal{R}$, such that $\nu(j)$ reflects in some sense the 'size' of $j$, with most indices being 'large':

$$
\nu(j) \geq 1 \text { for all } j \geq 0 \text { and } \#\{j: \nu(j) \leq K\}<\infty \text { for all } K>0 .
$$

We then define the analogues of higher empirical moments using the quantities $\nu_{r} \in \mathcal{R}$, defined by $\nu_{r}(j):=\nu(j)^{r}, r \geq 0$, setting

$$
S_{r}(X):=\sum_{j \geq 0} \nu_{r}(j) X^{j}, \quad X \in \mathcal{X}_{+},
$$

with, in particular, $S_{0}(X)=\|X\|_{1}$. Note that, because of (2.1), for any $r \geq 1$

$$
\#\left\{X \in \mathcal{X}_{+}: S_{r}(X) \leq K\right\}<\infty \text { for all } K>0
$$

To formulate the conditions that limit the growth of the empirical moments of $X_{N}(t)$ with $t$, we also define

$$
U_{r N}(X):=\sum_{J \in \mathcal{J}} \alpha^{J}\left(N^{-1} X\right) J^{T} \nu_{r} ; \quad V_{r N}(X):=\sum_{J \in \mathcal{J}} \alpha^{J}\left(N^{-1} X\right)\left(J^{T} \nu_{r}\right)^{2} .
$$


The assumptions that we shall need are then as follows.

Assumption 2.1 There exists a $\nu$ satisfying (2.1) and $r_{\max }^{(1)}, r_{\max }^{(2)} \geq 1$ such that, for all $X \in \mathcal{X}_{+}$,

$$
\sum_{J \in \mathcal{J}} \alpha^{J}\left(N^{-1} X\right)\left|J^{T} \nu_{r}\right|<\infty, \quad 0 \leq r \leq r_{\max }^{(1)}
$$

the case $r=0$ following from (1.1) and (1.2); furthermore, for some nonnegative constants $k_{r l}$, the inequalities

$$
\begin{aligned}
& N U_{0 N}(X) \leq k_{01} S_{0}(X)+N k_{04} \\
& N U_{1 N}(X) \leq k_{11} S_{1}(X)+N k_{14} \\
& N U_{r N}(X) \leq\left\{k_{r 1}+k_{r 2} N^{-1} S_{0}(X)\right\} S_{r}(X)+N k_{r 4}, \quad 2 \leq r \leq r_{\max }^{(1)}
\end{aligned}
$$

and

$$
\begin{aligned}
& N V_{0 N}(X) \leq k_{03} S_{1}(X)+N k_{05} \\
& N V_{r N}(X) \leq k_{r 3} S_{p(r)}(X)+N k_{r 5}, \quad 1 \leq r \leq r_{\max }^{(2)}
\end{aligned}
$$

are satisfied, where $1 \leq p(r) \leq r_{\max }^{(1)}$ for $1 \leq r \leq r_{\max }^{(2)}$.

The quantities $r_{\max }^{(1)}$ and $r_{\max }^{(2)}$ usually need to be reasonably large, if Assumption 4.2 below is to be satisfied.

Now, for $X_{N}$ as in the introduction, we let $t_{n}^{X_{N}}$ denote the time of its $n$-th jump, with $t_{0}^{X_{N}}=0$, and set $t_{\infty}^{X_{N}}:=\lim _{n \rightarrow \infty} t_{n}^{X_{N}}$, temporarily allowing for the possibility that $t_{\infty}^{X_{N}}$ may be finite. For $0 \leq t<t_{\infty}^{X_{N}}$, we define

$S_{r}^{(N)}(t):=S_{r}\left(X_{N}(t)\right) ; \quad U_{r}^{(N)}(t):=U_{r N}\left(X_{N}(t)\right) ; \quad V_{r}^{(N)}(t):=V_{r N}\left(X_{N}(t)\right)$,

and also

$$
\tau_{0}^{(N)}(C):=\inf \left\{t<t_{\infty}^{X_{N}}: S_{0}^{(N)}(t) \geq N C\right\}
$$

where the infimum of the empty set is taken to be $\infty$. Our first result limits the expectations of $S_{0}^{(N)}(t) \mathbf{1}_{\left[0, t_{\infty}^{X_{N}}\right)}(t)$ and $S_{1}^{(N)}(t) \mathbf{1}_{\left[0, t_{\infty}^{X_{N}}\right)}(t)$, for $t$ in some fixed interval $[0, T]$. Once it has been shown in Theorem 2.4 that $t_{\infty}^{X_{N}}=\infty$ a.s., we deduce that the bounds in fact hold for $\mathbf{E}\left\{S_{0}^{(N)}(t)\right\}$ and $\mathbf{E}\left\{S_{1}^{(N)}(t)\right\}$.

In what follows, we shall write $\mathcal{F}_{s}^{(N)}=\sigma\left(X_{N}(u), 0 \leq u \leq s\right)$, so that $\left(\mathcal{F}_{s}^{(N)}: s \geq 0\right)$ is the natural filtration of the process $X_{N}$. 
Lemma 2.2 Under Assumptions (2.5) and (2.6), for $0 \leq t \leq T$,

$$
\begin{aligned}
& \mathbf{E}\left\{S_{0}^{(N)}(t) \mathbf{1}_{\left[0, t_{\infty}^{X_{N}}\right)}(t)\right\} \leq\left(S_{0}^{(N)}(0)+N k_{04} t\right) e^{k_{01} t} \\
& \mathbf{E}\left\{S_{1}^{(N)}(t) \mathbf{1}_{\left[0, t_{\infty}^{X_{N}}\right)}(t)\right\} \leq\left(S_{1}^{(N)}(0)+N k_{14} t\right) e^{k_{11} t}
\end{aligned}
$$

Proof. For $l \in\{0,1\}$, define $M_{l}^{(N)}$ by

$$
M_{l}^{(N)}(t):=S_{l}^{(N)}(t)-S_{l}^{(N)}(0)-N \int_{0}^{t} U_{l}^{(N)}(u) d u, \quad 0 \leq t<t_{\infty}^{X_{N}} .
$$

It is immediate that $\left(M_{l}^{(N)}\left(t_{n}^{X_{N}}\right), n \geq 0\right)$ is a zero mean martingale with respect to $\left(\mathcal{F}_{t_{n}^{X_{N}}}^{(N)}: n \geq 0\right)$. Defining $N(s):=\inf \left\{m: t_{m}^{X_{N}} \geq s\right\}$, and using the martingale and Markov properties, it follows that

$$
\begin{aligned}
\mathbf{E}\left\{M_{l}^{(N)}\left(t_{N(s)}^{X_{N}}\right) I[N(s) \leq n]\right\} & =\mathbf{E}\left\{\mathbf{E}\left\{M_{l}^{(N)}\left(t_{N(s)}^{X_{N}}\right) I[N(s) \leq n] \mid \mathcal{F}_{s}^{(N)}\right\}\right\} \\
& =\mathbf{E}\left\{M_{l}^{(N)}(s) I[N(s) \leq n]\right\} .
\end{aligned}
$$

Applying the optional stopping theorem to the index $(N(s) \wedge n)$, we have that

$$
\mathbf{E}\left\{M_{l}^{(N)}\left(t_{N(s) \wedge n}^{X_{N}}\right)\right\}=0,
$$

in other words,

$$
\mathbf{E}\left\{M_{l}^{(N)}\left(t_{N(s)}^{X_{N}}\right) I[N(s) \leq n]\right\}+\mathbf{E}\left\{M_{l}^{(N)}\left(t_{n}^{X_{N}}\right) I[N(s)>n]\right\}=0 .
$$

But $N(s) \leq n$ if and only if $t_{n}^{X_{N}} \geq s$, so we deduce that

$$
\mathbf{E}\left\{M_{l}^{(N)}\left(s \wedge t_{n}^{X_{N}}\right)\right\}=0,
$$

for any $0 \leq s \leq T$ and any $n \geq 1$. This implies, using (2.6), that

$$
\begin{aligned}
\mathbf{E}\left\{S_{l}^{(N)}(t) \mathbf{1}_{[t, \infty)}\left(t_{n}^{X_{N}}\right)\right\} & \leq \mathbf{E}\left\{S_{l}^{(N)}\left(t \wedge t_{n}^{X_{N}}\right)\right\} \\
& =S_{l}^{(N)}(0)+\mathbf{E}\left\{\int_{0}^{t \wedge t_{n}^{X_{N}}}\left\{k_{l 1} S_{l}^{(N)}(u)+N k_{l 4}\right\} d u\right\} \\
& \leq S_{l}^{(N)}(0)+\int_{0}^{t}\left(k_{l 1} \mathbf{E}\left\{S_{l}^{(N)}\left(u \wedge t_{n}^{X_{N}}\right)\right\}+N k_{l 4}\right) d u
\end{aligned}
$$


Gronwall's inequality now implies that

$$
\mathbf{E}\left\{S_{l}^{(N)}\left(t \wedge t_{n}^{X}\right)\right\} \leq\left(S_{l}^{(N)}(0)+N k_{l 4} t\right) e^{k_{l 1} t},
$$

for any $n$, and the lemma follows from (2.12), by monotone convergence applied to variables $S_{l}^{(N)}(t) \mathbf{1}_{[t, \infty)}\left(t_{n}^{X_{N}}\right)$.

The next lemma shows that, if $C$ is large enough, then, with high probability, $N^{-1} S_{0}^{(N)}(t) \leq C$ holds for all $0 \leq t \leq T$ such that $t<t_{\infty}^{X_{N}}$. It is shown in Theorem 2.4 that $t_{\infty}^{X_{N}}=\infty$ a.s.

Lemma 2.3 Assume that Assumptions 2.1 are satisfied, and that $S_{0}^{(N)}(0) \leq$ $N C_{0}$ and $S_{1}^{(N)}(0) \leq N C_{1}$. Then, for any $C \geq 2\left(C_{0}+k_{04} T\right) e^{k_{01} T}$, we have

$$
\mathbf{P}\left[\left\{\tau_{0}^{(N)}(C) \leq T<t_{\infty}^{X_{N}}\right\}\right] \leq\left(C_{1} \vee 1\right) K_{00} /\left(N C^{2}\right),
$$

where $K_{00}$ depends on $T$ and the parameters of the model. Furthermore, $S_{0}^{(N)}(t)$ is almost surely bounded on $[0, T] \cap\left[0, t_{\infty}^{X_{N}}\right)$.

Proof. It is immediate from (2.10) and (2.6) that, for $0 \leq t<t_{\infty}^{X_{N}}$,

$$
\begin{aligned}
S_{0}^{(N)}(t) & =S_{0}^{(N)}(0)+N \int_{0}^{t} U_{0}^{(N)}(u) d u+M_{0}^{(N)}(t) \\
& \leq S_{0}^{(N)}(0)+\int_{0}^{t}\left(k_{01} S_{0}^{(N)}(u)+N k_{04}\right) d u+\sup _{0 \leq u \leq t} M_{0}^{(N)}(u) .
\end{aligned}
$$

Hence, from Gronwall's inequality, if $S_{0}^{(N)}(0) \leq N C_{0}$ and $0 \leq t<t_{\infty}^{X_{N}}$,

$$
S_{0}^{(N)}(t) \leq\left\{N\left(C_{0}+k_{04} T\right)+\sup _{0 \leq u \leq t} M_{0}^{(N)}(u)\right\} e^{k_{01} t} .
$$

Now, arguing as for (2.11), we have

$$
\mathbf{E}\left\{\left\{M_{0}^{(N)}\left(t \wedge t_{n}^{X_{N}}\right)\right\}^{2}-N \int_{0}^{t \wedge t_{n}^{X_{N}}} V_{0}^{(N)}(u) d u\right\}=0,
$$

from which it follows, much as above, that

$$
\begin{aligned}
\mathbf{E}\left(\left\{M_{0}^{(N)}\left(t \wedge t_{n}^{X_{N}}\right)\right\}^{2}\right) & \leq \mathbf{E}\left\{N \int_{0}^{t} V_{0}^{(N)}\left(u \wedge t_{n}^{X_{N}}\right) d u\right\} \\
& \leq \int_{0}^{t}\left\{k_{03} \mathbf{E} S_{1}^{(N)}\left(u \wedge t_{n}^{X_{N}}\right)+N k_{05}\right\} d u
\end{aligned}
$$


Using (2.13), we thus find that

$$
\mathbf{E}\left(\left\{M_{0}^{(N)}\left(t \wedge t_{n}^{X_{N}}\right)\right\}^{2}\right) \leq \frac{k_{03}}{k_{11}} N\left(C_{1}+k_{14} T\right)\left(e^{k_{11} t}-1\right)+N k_{05} t
$$

for each $n$. Hence $\left\{M_{0}^{(N)}\left(t \wedge t_{n}^{X_{N}}\right), n \geq 0\right\}$, is a square integrable martingale, implying that the limit $M_{0}^{(N)}\left(t \wedge t_{\infty}^{X_{N}}\right):=\lim _{n \rightarrow \infty} M_{0}^{(N)}\left(t \wedge t_{n}^{X_{N}}\right)$ is well defined, and satisfies $\mathbf{E} M_{0}^{(N)}\left(t \wedge t_{\infty}^{X_{N}}\right)=0$. Then, in view of the Markov property of $X_{N}$, it also follows that, for any $s \leq t$,

$$
\mathbf{E}\left\{M_{0}^{(N)}\left(t \wedge t_{\infty}^{X_{N}}\right) \mid \mathcal{F}_{s}^{(N)}\right\}=M_{0}^{(N)}\left(s \wedge t_{\infty}^{X_{N}}\right),
$$

where, as before, $\mathcal{F}_{s}^{(N)}:=\sigma\left\{X_{N}(u), 0 \leq u \leq s\right\}$, so that $\left\{M_{0}^{(N)}\left(s \wedge t_{\infty}^{X_{N}}\right), 0 \leq s \leq t\right\}$ is also a square integrable martingale. This in turn, by the martingale convergence theorem, implies from (2.15) that

$$
\sup _{0 \leq u<t \wedge t_{\infty}^{X_{N}}} S_{0}^{(N)}(u)<\infty
$$

for any $t$.

Doob's maximal inequality now allows us to deduce that, for any $a>0$,

$$
\begin{aligned}
\mathbf{P}\left[\sup _{0 \leq u \leq T} M_{0}^{(N)}\left(u \wedge t_{\infty}^{X_{N}}\right)>a N\right] & \leq \mathbf{P}\left[\sup _{0 \leq n \leq \infty} M_{0}^{(N)}\left(T \wedge t_{n}^{X_{N}}\right)>a N\right] \\
& \leq \frac{1}{N a^{2}}\left\{\frac{k_{03}}{k_{11}}\left(C_{1}+k_{14} T\right)\left\{e^{k_{11} T}-1\right\}+k_{05} T\right\} \\
& =: \frac{C_{1} K_{01}+K_{02}}{N a^{2}} .
\end{aligned}
$$

Taking $a=\frac{1}{2} C e^{-k_{01} T}$ and putting the result into (2.15), the lemma follows.

As a consequence, $S_{0}^{(N)}(t)=\left\|X_{N}(t)\right\|_{1}$ is almost surely bounded up to $t_{\infty}^{X_{N}}$ if $t_{\infty}^{X_{N}}<\infty$. This does not of itself imply that the event $t_{\infty}^{X_{N}}<\infty$ has zero probability, because the transition rates out of sets of states $X$ with $\|X\|_{1}$ bounded may still be unbounded. In the following theorem, we show that $\mathbf{P}\left[t_{\infty}^{X_{N}}<\infty\right]$ is in fact 0 , and control the 'higher $\nu$-moments' $S_{r}^{(N)}(t)$ of $X_{N}(t)$. 
Theorem 2.4 Suppose that conditions of Lemma 2.3 hold. Assume also that $S_{1}^{(N)}(0) \leq N C_{1}$ and $S_{p(1)}^{(N)}(0) \leq N C_{1}^{\prime}$. Then $\mathbf{P}\left[t_{\infty}^{X_{N}}<\infty\right]=0$. Also, for $2 \leq r \leq r_{\max }^{(1)}$ and for any $C>0$, we have

$$
\mathbf{E}\left\{S_{r}^{(N)}\left(t \wedge \tau_{0}^{(N)}(C)\right)\right\} \leq\left(S_{r}^{(N)}(0)+N k_{r 4} t\right) e^{\left(k_{r 1}+C k_{r 2}\right) t}, \quad 0 \leq t \leq T .
$$

Furthermore, if for $1 \leq r \leq r_{\max }^{(2)}, S_{r}^{(N)}(0) \leq N C_{r}$ and $S_{p(r)}^{(N)}(0) \leq N C_{r}^{\prime}$, then, for any $\gamma \geq 1$,

$$
\mathbf{P}\left[\sup _{0 \leq t \leq T} S_{r}^{(N)}\left(t \wedge \tau_{0}^{(N)}(C)\right) \geq N \gamma C_{r T}^{\prime \prime}\right] \leq K_{r 0} \gamma^{-2} N^{-1},
$$

where

$$
C_{r T}^{\prime \prime}:=\left(C_{r}+k_{r 4} T+\sqrt{\left(C_{r}^{\prime} \vee 1\right)}\right) e^{\left(k_{r 1}+C k_{r 2}\right) T}
$$

and $K_{r 0}$ depends on $C, T$ and the parameters of the model.

Proof. Defining

$$
M_{r}^{(N)}(t):=S_{r}^{(N)}(t)-S_{r}^{(N)}(0)-N \int_{0}^{t} U_{r}^{(N)}(u) d u, \quad 0 \leq t<t_{\infty}^{X_{N}},
$$

as in (2.10), but now for any $r \in \mathbb{Z}_{+}$, it follows as for (2.11) that

$$
\mathbf{E} M_{r}^{(N)}\left(t \wedge t_{n}^{X_{N}} \wedge \tau_{0}^{(N)}(C)\right)=0
$$

for all $t, n, r$. Arguing as before, this, together with (2.6), implies that, if $2 \leq r \leq r_{\max }^{(1)}$, then

$$
\begin{aligned}
& \mathbf{E} S_{r}^{(N)}\left(t \wedge t_{n}^{X_{N}} \wedge \tau_{0}^{(N)}(C)\right) \\
& \quad \leq S_{r}^{(N)}(0)+\int_{0}^{t}\left(\left\{k_{r 1}+C k_{r 2}\right\} \mathbf{E}\left\{S_{r}^{(N)}\left(u \wedge t_{n}^{X_{N}} \wedge \tau_{0}^{(N)}(C)\right)\right\}+N k_{r 4}\right) d u .
\end{aligned}
$$

Gronwall's inequality now implies that, for $2 \leq r \leq r_{\max }^{(1)}$,

$$
\mathbf{E} S_{r}^{(N)}\left(t \wedge t_{n}^{X_{N}} \wedge \tau_{0}^{(N)}(C)\right) \leq\left(S_{r}^{(N)}(0)+N k_{r 4} t\right) e^{\left(k_{r 1}+C k_{r 2}\right) t} .
$$

Now, also from (2.21) and (2.6), we have, for $t<t_{\infty}^{X_{N}}$ and each $r$,

$$
\begin{aligned}
& S_{r}^{(N)}\left(t \wedge \tau_{0}^{(N)}(C)\right) \\
& =S_{r}^{(N)}(0)+N \int_{0}^{t \wedge \tau_{0}^{(N)}(C)} U_{r}^{(N)}(u) d u+M_{r}^{(N)}\left(t \wedge \tau_{0}^{(N)}(C)\right) \\
& \leq S_{r}^{(N)}(0)+\int_{0}^{t}\left(\left\{k_{r 1}+C k_{r 2}\right\} S_{r}^{(N)}\left(u \wedge \tau_{0}^{(N)}(C)\right)+N k_{r 4}\right) d u \\
& \quad+\sup _{0 \leq u \leq t} M_{r}^{(N)}\left(u \wedge \tau_{0}^{(N)}(C)\right) ;
\end{aligned}
$$


for $r=1$, we interpret $k_{12}=0$. Hence, from Gronwall's inequality,

$$
S_{r}^{(N)}\left(t \wedge \tau_{0}^{(N)}(C)\right) \leq\left\{N\left(C_{r}+k_{r 4} t\right)+\sup _{0 \leq u \leq t} M_{r}^{(N)}\left(u \wedge \tau_{0}^{(N)}(C)\right)\right\} e^{\left(k_{r 1}+C k_{r 2}\right) t}
$$

whenever $t<t_{\infty}^{X_{N}}$, for all $r$.

Now, as in (2.16), we have, for each $r$,

$$
\mathbf{E}\left\{\left\{M_{r}^{(N)}\left(t \wedge t_{n}^{X_{N}} \wedge \tau_{0}^{(N)}(C)\right)\right\}^{2}-N \int_{0}^{t \wedge t_{n}^{X_{N}} \wedge \tau_{0}^{(N)}(C)} V_{r}^{(N)}(u) d u\right\}=0
$$

from which it follows, using $(2.7)$, that, for $1 \leq r \leq r_{\max }^{(2)}$,

$$
\begin{aligned}
\mathbf{E} & \left(\left\{M_{r}^{(N)}\left(t \wedge t_{n}^{X_{N}} \wedge \tau_{0}^{(N)}(C)\right)\right\}^{2}\right) \\
& \leq \mathbf{E}\left\{N \int_{0}^{\left.t \wedge t_{n}^{X_{N}} \wedge \tau_{0}^{(N)}(C)\right)} V_{r}^{(N)}(u) d u\right\} \\
& \leq \int_{0}^{t}\left\{k_{r 3} \mathbf{E} S_{p(r)}^{(N)}\left(u \wedge t_{n}^{X_{N}} \wedge \tau_{0}^{(N)}(C)\right)+N k_{r 5}\right\} d u \\
& \leq \frac{N\left(C_{r}^{\prime}+k_{p(r), 4} T\right) k_{r 3}}{k_{p(r), 1}+C k_{p(r), 2}}\left(e^{\left(k_{p(r), 1}+C k_{p(r), 2} t\right)}-1\right)+N k_{p(r), 5} T,
\end{aligned}
$$

this last by $(2.22)$, since $p(r) \leq r_{\max }^{(1)}$ for $1 \leq r \leq r_{\max }^{(2)}$.

Thus, once again, $M_{r}^{(N)}\left(t \wedge t_{\infty}^{X_{N}} \wedge \tau_{0}^{(N)}(C)\right)$, defined to be $\lim _{n \rightarrow \infty} M_{r}^{(N)}(t \wedge$ $\left.t_{n}^{X_{N}} \wedge \tau_{0}^{(N)}(C)\right)$, is a square integrable martingale in $t$, and $\sup _{0 \leq t \leq T} M_{r}^{(N)}(t \wedge$ $\left.t_{\infty}^{X_{N}} \wedge \tau_{0}^{(N)}(C)\right)$ is almost surely finite. It thus follows from (2.23) and Lemma 2.3 that, in particular,

$$
\sup _{0 \leq t<T \wedge t_{\infty}^{X_{N}}} S_{1}^{(N)}(t)<\infty
$$

for any $T$, which then, from (2.3) and (1.2), in turn implies that $\mathbf{P}\left[t_{\infty}^{X_{N}} \leq\right.$ $T]=0$ for all $T$.

It is now immediate from (2.22) and Fatou's lemma that (2.19) holds. Finally, Doob's inequality implies that, for any $a>0$,

$$
\begin{aligned}
\mathbf{P} & {\left[\sup _{0 \leq u \leq T} M_{r}^{(N)}\left(u \wedge \tau_{0}^{(N)}(C)\right)>a N\right] } \\
& \leq \frac{1}{N a^{2}}\left\{\frac{k_{r 3}\left(C_{r}^{\prime}+k_{p(r), 4} T\right)}{k_{p(r), 1}+C k_{p(r), 2}}\left(e^{\left(k_{p(r), 1}+C k_{p(r), 2} T\right)}-1\right)+k_{p(r), 5} T\right\}
\end{aligned}
$$




$$
=: \frac{C_{r}^{\prime} K_{r 1}+K_{r 2}}{N a^{2}} .
$$

Taking $a=\gamma \sqrt{\left(C_{r}^{\prime} \vee 1\right)}$ and putting the result into (2.23), we have proved (2.20), with $K_{r 0}=\left(C_{r}^{\prime} K_{r 1}+K_{r 2}\right) /\left(C_{r}^{\prime} \vee 1\right)$.

Note also that it follows, from Lemma 2.3 and Theorem 2.4, that $\sup _{0 \leq t \leq T} S_{r}^{(N)}(t)<$ $\infty$ a.s. for all $0 \leq r \leq r_{\max }^{(2)}$.

In what follows, we shall particularly need to control quantities of the form $\sum_{J \in \mathcal{J}} \alpha^{J}\left(x_{N}(s)\right) d(J, \zeta)$, where $x_{N}:=N^{-1} X_{N}$ and

$$
d(J, \zeta):=\sum_{j \geq 0}\left|J^{j}\right| \zeta(j)
$$

for $\zeta \in \mathcal{R}$ chosen such that $\zeta(j) \geq 1$ grows fast enough with $j$. Defining

$$
\tau^{(N)}(a, \zeta):=\inf \left\{s: \sum_{J \in \mathcal{J}} \alpha^{J}\left(x_{N}(s)\right) d(J, \zeta) \geq a\right\},
$$

we show in the following corollary that, under suitable assumptions, $\tau^{(N)}(a, \zeta)$ is rarely less than $T$.

Corollary 2.5 Suppose that conditions of Lemma 2.3 hold, and that $\zeta$ is such that

$$
\sum_{J \in \mathcal{J}} \alpha^{J}\left(N^{-1} X\right) d(J, \zeta) \leq\left\{k_{1} N^{-1} S_{r}(X)+k_{2}\right\}^{b}
$$

for some $1 \leq r:=r(\zeta) \leq r_{\max }^{(2)}$ and some $b=b(\zeta) \geq 1$. For this value of $r$, assume that $S_{r}^{(N)}(0) \leq N C_{r}$ and $S_{p(r)}^{(N)}(0) \leq N C_{r}^{\prime}$ for some constants $C_{r}$ and $C_{r}^{\prime}$. Assume further that $S_{0}^{(N)}(0) \leq N C_{0}, S_{1}^{(N)}(0) \leq N C_{1}$ for some constants $C_{0}, C_{1}$, and define $C:=2\left(C_{0}+k_{04} T\right) e^{k_{01} T}$. Then

$$
\mathbf{P}\left[\tau^{(N)}(a, \zeta) \leq T\right] \leq N^{-1}\left\{K_{r 0} \gamma_{a}^{-2}+K_{00}\left(C_{1} \vee 1\right) C^{-2}\right\},
$$

for any $a \geq\left\{k_{2}+k_{1} C_{r T}^{\prime \prime}\right\}^{b}$, where $\gamma_{a}:=\left(a^{1 / b}-k_{2}\right) /\left\{k_{1} C_{r T}^{\prime \prime}\right\}, K_{r 0}$ and $C_{r T}^{\prime \prime}$ are as in Theorem 2.4, and $K_{00}$ is as in Lemma 2.3.

Proof. In view of (2.27), it is enough to bound the probability

$$
\mathbf{P}\left[\sup _{0 \leq t \leq T} S_{r}^{(N)}(t) \geq N\left(a^{1 / b}-k_{2}\right) / k_{1}\right] .
$$


However, Lemma 2.3 and Theorem 2.4 together bound this probability by

$$
N^{-1}\left\{K_{r 0} \gamma_{a}^{-2}+K_{00}\left(C_{1} \vee 1\right) C^{-2}\right\}
$$

where $\gamma_{a}$ is as defined above, as long as $a^{1 / b}-k_{2} \geq k_{1} C_{r T}^{\prime \prime}$.

Under the conditions of the corollary, it follows that $\sum_{J \in \mathcal{J}} \alpha^{J}\left(x_{N}(s)\right) d(J, \zeta)$ is a.s. bounded on $0 \leq s \leq T$, and that it is bounded by $\left\{k_{2}+k_{1} C_{r, T}^{\prime \prime}\right\}^{b}$, except on an event of probability of order $O\left(N^{-1}\right)$. Usually, one can choose $b=1$.

\section{$3 \quad$ Semigroup properties}

We make the following initial assumption about the matrix $A$ :

$$
A_{i j} \geq 0 \text { for all } i \neq j \geq 0 ; \quad \sum_{j \neq i} A_{j i}<\infty \text { for all } i \geq 0
$$

We then define $Y$ to be a Markov branching process with countably many types $i=0,1,2, \ldots$, related to the matrix $A$ in the following way. A type $i$ individual has death rate $d_{i}$ given by

$$
d_{i}:=\left|A_{i i}\right|+\sum_{j \neq i} A_{i j}^{T}<\infty,
$$

finite by assumption; at death, the $i$-individual is replaced by

$2 \quad$ type $i$ individuals with probability $\frac{A_{i i}^{+}}{d_{i}}$;

$1 \quad$ type $i$ and 1 type $j$ individual with probability $\frac{A_{i j}^{T}}{d_{i}}$;

$0 \quad$ individuals with probability $\frac{A_{i i}^{-}}{d_{i}}$,

where $A_{i i}^{+}:=\max \left\{A_{i i}, 0\right\}$ and $A_{i i}^{-}:=-\min \left\{A_{i i}, 0\right\}$. We then define the family of matrices $R:=\left\{R_{i j}(t), t \geq 0\right\}$ by

$$
R_{i j}(t):=\mathbf{E}_{i} Y^{j}(t):=\mathbf{E}\left\{Y^{j}(t) \mid Y(0)=e^{(i)}\right\}
$$


Clearly, by conditioning on the value of $Y(s)$, we see that $R(s+t)=R(s) R(t)$, so that the matrices form a semigroup. Under the further mild condition, that

$$
A^{T} \mu \leq w \mu \text { for some } w \geq 0 \text { and } \mu \in R_{+}^{\mathbb{Z}_{+}} \text {such that } \mu(m) \geq 1, m \geq 0,
$$

we can deduce a bound on the rate of growth of the elements $R_{i j}(t)$ with respect to the $\mu$-norm

$$
\|\xi\|_{\mu}:=\sum_{m \geq 0} \mu(m)\left|\xi^{m}\right| \quad \text { on } \quad \mathcal{R}_{\mu}:=\left\{\xi \in \mathcal{R}:\|\xi\|_{\mu}<\infty\right\} .
$$

Theorem 3.1 Let A satisfy Assumptions (3.1) and (3.2). Then $R^{T}$ is a strongly continuous semigroup on $\mathcal{R}_{\mu}$, with generator $A$. Furthermore,

$$
\sum_{j \geq 0} R_{i j}(t) \mu(j) \leq \mu(i) e^{w t} \quad \text { for all } i \text { and } t .
$$

Proof. We begin with the Feller backward recursion for $R(t)$. We define $Y_{j}^{\langle n\rangle}(t)$ to be the number of type $j$ individuals alive at time $t$ that were born at most $n$ generations after the original ancestor, and we set

$$
R_{i j}^{\langle n\rangle}(t):=\mathbf{E}_{i}\left\{Y_{j}^{\langle n\rangle}(t)\right\}
$$

Then it is clear that the inequality

$$
\sum_{j \geq 0} R_{i j}^{\langle n\rangle}(t) \mu(j) \leq \mu(i) e^{w t}, \quad i \geq 0, t \geq 0,
$$

is true if $n=0$, since then the left hand side is just $\mu(i) e^{-d_{i} t}$, and $w \geq 0$. Now, by conditioning on the first event, we have

$$
\begin{aligned}
& R_{i j}^{\langle n+1\rangle}(t)=\delta_{i j} e^{-d_{i} t} \\
& \quad+\int_{0}^{t} d_{i} e^{-d_{i} u}\left\{\sum_{l \neq i} \frac{A_{i l}^{T}}{d_{i}}\left[R_{l j}^{\langle n\rangle}(t-u)+R_{i j}^{\langle n\rangle}(t-u)\right]+2 \frac{A_{i i}^{+}}{d_{i}} R_{i j}^{\langle n\rangle}(t-u)\right\} d u .
\end{aligned}
$$

If (3.5) holds for $n$, then the right hand side of (3.6) can be bounded above, giving

$$
\sum_{j \geq 0} R_{i j}^{\langle n+1\rangle}(t) \mu(j)
$$




$$
\begin{aligned}
& \leq \mu(i) e^{-d_{i} t}+\int_{0}^{t} e^{-d_{i} u}\left\{\sum_{l \neq i} A_{i l}^{T}[\mu(l)+\mu(i)]+2 A_{i i}^{+} \mu(i)\right\} e^{w(t-u)} d u \\
& \leq e^{w t}\left\{\mu(i) e^{-\left(d_{i}+w\right) t}+\frac{\kappa_{i}}{d_{i}+w}\left(1-e^{-\left(d_{i}+w\right) t}\right)\right\}
\end{aligned}
$$

where

$$
\kappa_{i}:=\sum_{l \neq i} A_{i l}^{T}[\mu(l)+\mu(i)]+2 A_{i i}^{+} \mu(i) .
$$

Hence (3.5) also holds for $n+1$, provided that $\kappa_{i} /\left(d_{i}+w\right) \leq \mu(i)$ for all $i$; but this is equivalent to the condition $\sum_{l \neq i} A_{i l}^{T} \mu(l)+\mu(i)\left\{2 A_{i i}^{+}-\left|A_{i i}\right|\right\} \leq w \mu(i)$ for all $i$, which, by considering the cases $A_{i i} \geq 0$ and $A_{i i}<0$ in turn, is just the inequality $A^{T} \mu \leq w \mu$ that we have assumed given. Letting $n$ tend to infinity in $(3.5), R_{i j}^{\langle n\rangle}(t)$ increases to $R_{i j}(t)$ (as defined by the minimal process), and we have shown that (3.4) holds.

Then, returning to (3.6), we can let $n \rightarrow \infty$, which by monotone convergence yields

$$
\begin{aligned}
R_{i j}(t) & =\delta_{i j} e^{-d_{i} t} \\
& +\int_{0}^{t} e^{-d_{i} u}\left\{\sum_{l \neq i} A_{i l}^{T}\left[R_{l j}(t-u)+R_{i j}(t-u)\right]+2 A_{i i}^{+} R_{i j}(t-u)\right\} d u
\end{aligned}
$$

with the sums all absolutely convergent in view of (3.4). From this, it follows that

$$
\begin{aligned}
& e^{d_{i} t} R_{i j}(t) \\
& \quad=\delta_{i j}+\int_{0}^{t} e^{d_{i} v}\left\{\sum_{l \neq i} A_{i l}^{T}\left[R_{l j}(v)+R_{i j}(v)\right]+2 A_{i i}^{+} R_{i j}(v)\right\} d v
\end{aligned}
$$

This equation shows immediately that each $R_{i j}(t)$ is a continuous function of $t$, because an indefinite integral has to be; in particular, at $t=0$, we thus have

$$
\lim _{t \rightarrow 0} R_{i j}(t)=\delta_{i j}
$$

Then since, from (3.4), $R_{l j}(v) \leq \mu(l) e^{w v} / \mu(j)$, it follows that the sum $\sum_{l \neq i} A_{i l}^{T} R_{l j}(v)$ is uniformly convergent, and hence is also continuous. Thus the indefinite 
integral is differentiable, and the integrand is its derivative. Hence we have shown that

$$
\begin{aligned}
d_{i} R_{i j}(t)+\frac{d}{d t} R_{i j}(t) & =\sum_{l \neq i} A_{i l}^{T}\left[R_{l j}(t)+R_{i j}(t)\right]+2 A_{i i}^{+} R_{i j}(t) \\
& =\sum_{l \neq i} A_{i l}^{T} R_{l j}(t)+\left(d_{i}+A_{i i}\right) R_{i j}(t),
\end{aligned}
$$

and thus

$$
\frac{d}{d t} R_{i j}(t)=\left(A^{T} R(t)\right)_{i j} ; \quad \frac{d}{d t} R_{i j}(0)=A_{i j}^{T},
$$

for all $i, j$. Note also that, from (3.4),

$$
\limsup _{t \rightarrow 0} \sum_{j \geq 0} R_{i j}(t) \mu(j) \leq \mu(i) \lim _{t \rightarrow 0} e^{w t}=\mu(i)
$$

whereas

$$
\liminf _{t \rightarrow 0} \sum_{j \geq 0} R_{i j}(t) \mu(j) \geq \lim _{t \rightarrow 0} R_{i i}(t) \mu(i)=\mu(i)
$$

so that

$$
\lim _{t \rightarrow 0} \sum_{j \geq 0} R_{i j}(t) \mu(j)=\mu(i) ; \quad \lim _{t \rightarrow 0} \sum_{j \neq i} R_{i j}(t) \mu(j)=0 .
$$

To show that $R^{T}$ is the semigroup on $\mathcal{R}_{\mu}$ generated by $A$, we need rather more. First, we observe that, for $\xi \in \mathcal{R}_{\mu}$,

$$
\left\|\xi^{T} R(t)\right\|_{\mu} \leq \sum_{j \geq 0} \mu(j) \sum_{i \geq 0}\left|\xi^{i}\right| R_{i j}(t) \leq \sum_{i \geq 0}\left|\xi^{i}\right| \mu(i) e^{w t}=\|\xi\|_{\mu} e^{w t}
$$

by (3.4), so that $R$ indeed maps $\mathcal{R}_{\mu}$ into itself. Then, to show strong continuity, we note that, for $\xi \in \mathcal{R}_{\mu}$,

$$
\begin{aligned}
\left\|\xi^{T} R(t)-\xi^{T}\right\|_{\mu} \leq & \sum_{j \geq 0} \mu(j)\left\{\sum_{i \neq j}\left|\xi^{i}\right| R_{i j}(t)+\left|\xi^{j}\right|\left|R_{j j}(t)-1\right|\right\} \\
= & \sum_{i \geq 0}\left|\xi^{i}\right| \sum_{j \neq i} R_{i j}(t) \mu(j) \\
& +\sum_{j \geq 0} \mu(j)\left|\xi^{j}\right|\left|R_{j j}(t)-1\right| .
\end{aligned}
$$


It is immediate from (3.4) that $\left|R_{j j}(t)-1\right| \leq e^{w t}$, so that dominated convergence can be used as $t \rightarrow 0$ in (3.14), giving the limit 0 , in view of (3.9). Then

$$
0 \leq \sum_{j \neq i} R_{i j}(t) \mu(j) \leq \mu(i) e^{w t},
$$

by (3.4), and since $\xi \in \mathcal{R}_{\mu}$ we can also use dominated convergence in (3.13); by (3.11), this then also yields 0 . Hence, for $\xi \in \mathcal{R}_{\mu}$,

$$
\lim _{t \rightarrow 0}\left\|\xi^{T} R(t)-\xi^{T}\right\|_{\mu}=0
$$

proving strong continuity.

To show that $R^{T}$ is generated by $A$, we now need to show that

$$
\lim _{t \rightarrow 0} t^{-1}\left\|\xi^{T} R(t)-\xi^{T}-t \xi^{T} A^{T}\right\|_{\mu}=0
$$

for $\xi$ in a dense subset of $\mathcal{R}_{\mu}$. Taking first $\xi=e^{(i)}$, the $i$-th coordinate vector, we need to examine

$$
t^{-1} \sum_{j \geq 0} \mu(j)\left|R_{i j}(t)-\delta_{i j}-t A_{i j}^{T}\right|
$$

From (3.7), and again noting that $\sum_{l \neq i} A_{i l}^{T}+2 A_{i i}^{+}=d_{i}+A_{i i}$, we have

$$
\begin{aligned}
R_{i j}(t) & -\delta_{i j}-t A_{i j}^{T} \\
= & \int_{0}^{t}\left(e^{-d_{i} u}\left\{\sum_{l \neq i} A_{i l}^{T} R_{l j}(t-u)+\left(d_{i}+A_{i i}\right) R_{i j}(t-u)-d_{i} \delta_{i j}\right\}-A_{i j}^{T}\right) d u \\
= & \int_{0}^{t} e^{-d_{i} u}\left\{\sum_{l \geq 0} A_{i l}^{T}\left(R_{l j}(t-u)-\delta_{l j}\right)+d_{i}\left(R_{i j}(t-u)-\delta_{i j}\right)\right\} d u \\
& \quad-A_{i j}^{T} \int_{0}^{t}\left(1-e^{-d_{i} u}\right) d u .
\end{aligned}
$$

Hence it follows directly that

$$
\begin{aligned}
& t^{-1} \sum_{j \geq 0} \mu(j)\left|R_{i j}(t)-\delta_{i j}-t A_{i j}^{T}\right| \\
& \quad \leq t^{-1} \sum_{j \geq 0} \mu(j) \int_{0}^{t}\left\{d_{i}\left\{R_{i j}(v)\left(1-\delta_{i j}\right)+\delta_{i j}\left|R_{i i}(v)-1\right|\right\}\right.
\end{aligned}
$$




$$
\begin{gathered}
\left.+\sum_{l \neq j}\left|A_{i l}^{T}\right| R_{l j}(v)+\left|A_{i j}^{T}\right|\left|1-R_{i i}(v)\right|\right\} d v \\
+\sum_{j \geq 0} \mu(j)\left|A_{i j}^{T}\right| t^{-1} \int_{0}^{t}\left(1-e^{-d_{i} u}\right) d u \\
\leq d_{i} f_{1 i}(t)+\mu(i) f_{2 i}(t)+\sum_{l \geq 0}\left|A_{i l}^{T}\right| f_{1 l}(t) \\
+\sum_{j \geq 0}\left|A_{i j}^{T}\right| \mu(j) f_{2 j}(t)+\frac{t d_{i}}{2} \sum_{j \geq 0}\left|A_{i j}^{T}\right| \mu(j),
\end{gathered}
$$

where

$$
f_{1 l}(t):=\sup _{0 \leq v \leq t} \sum_{j \neq l} R_{l j}(v) \mu(j) ; \quad f_{2 l}(t):=\sup _{0 \leq v \leq t}\left|1-R_{l l}(v)\right| .
$$

Now

$$
\lim _{t \rightarrow 0} f_{1 l}(t)=\lim _{t \rightarrow 0} f_{2 l}(t)=0,
$$

by (3.11) and (3.9), and

$$
f_{1 l}(t) \leq \mu(l) e^{w t} ; \quad f_{2 l}(t) \leq e^{w t},
$$

in view of (3.4); furthermore,

$$
\sum_{l \geq 0}\left|A_{i l}^{T}\right| \mu(l)=\sum_{l \geq 0} A_{i l}^{T} \mu(l)+\left(\left|A_{i i}\right|-A_{i i}\right) \mu(i) \leq\left(w+2\left|A_{i i}\right|\right) \mu(i)<\infty .
$$

These observations and dominated convergence now show that the right hand side of (3.17) converges to zero as $t \rightarrow 0$.

We have now shown that (3.15) is satisfied for $\xi=e^{(i)}$, for any $i$. The same argument shows that (3.15) is satisfied for arbitrary finite linear combinations of the $e^{(i)}$, and a density argument completes the proof.

Remark. Note that there may be many possible choices for $\mu$. In what follows, it is important that $F$ be a Lipschitz operator with respect to the $\mu$-norm, and this has to be borne in mind when choosing $\mu$.

\section{Main approximation}

Let $X_{N}, N \geq 1$, be a sequence of pure jump Markov processes as in Section 1 , with $A$ and $F$ defined as in (1.3) and (1.4), and suppose that $F: \mathcal{R}_{\mu} \rightarrow \mathcal{R}_{\mu}$, 
with $\mathcal{R}_{\mu}$ as defined in (3.3), for some $\mu$ such that Assumption (3.2) holds. Suppose also that $F$ is locally Lipschitz in the $\mu$-norm: for any $z>0$,

$$
\sup _{x \neq y:\|x\|_{\mu},\|y\|_{\mu} \leq z}\|F(x)-F(y)\|_{\mu} /\|x-y\|_{\mu} \leq K(\mu, F ; z)<\infty .
$$

Then, for $x(0) \in \mathcal{R}_{\mu}$, the integral equation

$$
x(t)=R^{T}(t) x(0)+\int_{0}^{t} R^{T}(t-s) F(x(s)) d s .
$$

has a unique continuous solution $x$ in $\mathcal{R}_{\mu}$ on some non-empty time interval $\left[0, t_{\max }\right)$, such that, if $t_{\max }<\infty$, then $\|x(t)\|_{\mu} \rightarrow \infty$ as $t \rightarrow t_{\max }$ (Pazy 1983, Theorem 1.4, Chapter 6), and $x$ represents a mild solution of the deterministic equations (1.3). We now wish to show that the process $x_{N}:=N^{-1} X_{N}$ is close to $x$. To do so, we need a corresponding representation for $X_{N}$.

To find such a representation, let $W(t), t \geq 0$, be a pure jump path on $\mathcal{X}_{+}$ that has only finitely many jumps up to time $T$. Then we can write

$$
W(t)=W(0)+\sum_{j: \sigma_{j} \leq t} \Delta W\left(\sigma_{j}\right), \quad 0 \leq t \leq T,
$$

where $\Delta W(s):=W(s)-W(s-)$ and $\sigma_{j}, j \geq 1$, denote the times when $W$ has its jumps. Now let $A^{T}$ be a mean intensity matrix satisfying the conditions of Theorem 3.1, and let $R(\cdot)$ be the associated semigroup. Define the path $W^{*}(t), 0 \leq t \leq T$, from the equation

$$
W^{*}(t):=R^{T}(t) W(0)+\sum_{j: \sigma_{j} \leq t} R^{T}\left(t-\sigma_{j}\right) \Delta_{j}-\int_{0}^{t} R^{T}(t-s) A W(s) d s,
$$

where $\Delta_{j}:=\Delta W\left(\sigma_{j}\right)$. Note that the latter integral makes sense, because each of the sums $\sum_{j \geq 0} R_{i j}^{T} A_{j k}, k \geq 0$, is well defined, since $R$ is non-negative and $A$ is non-negative off the diagonal, and because only finitely many of the coordinates of $W$ are non-zero.

Lemma $4.1 W^{*}(t)=W(t)$ for all $0 \leq t \leq T$.

Proof. Fix any $t$, and suppose that $W^{*}(s)=W(s)$ for all $s \leq t$. This is clearly the case for $t=0$. Let $\sigma(t)>t$ denote the time of the first jump 
of $W$ after $t$. Then, for any $0<h<\sigma(t)-t$, using the semigroup property for $R$ and (4.4),

$$
\begin{aligned}
& W^{*}(t+h)-W^{*}(t) \\
& =\left(R^{T}(h)-I\right) R^{T}(t) W(0)+\sum_{j: \sigma_{j} \leq t}\left(R^{T}(h)-I\right) R^{T}\left(t-\sigma_{j}\right) \Delta_{j} \\
& \quad-\int_{0}^{t}\left(R^{T}(h)-I\right) R^{T}(t-s) A W(s) d s-\int_{t}^{t+h} R^{T}(t+h-s) A W(t) d s,
\end{aligned}
$$

where, in the last integral, we use the fact that there are no jumps of $W$ between $t$ and $t+h$. Thus we have

$$
\begin{aligned}
& W^{*}(t+h)-W^{*}(t) \\
& =\left(R^{T}(h)-I\right)\left\{R^{T}(t) W(0)+\sum_{j: \sigma_{j} \leq t} R^{T}\left(t-\sigma_{j}\right) \Delta_{j}-\int_{0}^{t} R^{T}(t-s) A W(s) d s\right\} \\
& \quad-\int_{t}^{t+h} R^{T}(t+h-s) A W(t) d s \\
& =\left(R^{T}(h)-I\right) W(t)-\int_{t}^{t+h} R^{T}(t+h-s) A W(t) d s .
\end{aligned}
$$

But now, since $A^{T}$ is the infinitesimal matrix associated with $R$, we have

$$
\int_{t}^{t+h} R^{T}(t+h-s) A x d s=\left(R^{T}(h)-I\right) x
$$

for all $x \in \mathcal{X}_{+}$, so that $W^{*}(t+h)=W^{*}(t)$ for all $t+h<\sigma(t)$, implying that $W^{*}(s)=W(s)$ for all $s<\sigma(t)$. On the other hand, from $(4.4), W^{*}(\sigma(t))-$ $W^{*}(\sigma(t)-)=\Delta W(\sigma(t))$, so that $W^{*}(s)=W(s)$ for all $s \leq \sigma(t)$. Thus we can prove equality over the interval $\left[0, \sigma_{1}\right]$, and then successively over the intervals $\left[\sigma_{j}, \sigma_{j+1}\right]$, until $[0, T]$ is covered.

Now suppose that $W$ arises as a realization of $X_{N}$. Then $X_{N}$ has transition rates such that

$$
M_{N}(t):=\sum_{j: \sigma_{j} \leq t} \Delta X_{N}\left(\sigma_{j}\right)-\int_{0}^{t} A X_{N}(s) d s-\int_{0}^{t} N F\left(x_{N}(s)\right) d s
$$


is a zero mean local martingale. In view of Lemma 4.1, we can use (4.4) to write

$$
X_{N}(t)=R^{T}(t) X_{N}(0)+\widetilde{M}_{N}(t)+N \int_{0}^{t} R^{T}(t-s) F\left(x_{N}(s)\right) d s
$$

where

$$
\begin{aligned}
\widetilde{M}_{N}(t): & \sum_{j: \sigma_{j} \leq t} R^{T}\left(t-\sigma_{j}\right) \Delta X_{N}\left(\sigma_{j}\right) \\
& -\int_{0}^{t} R^{T}(t-s) A X_{N}(s) d s-\int_{0}^{t} R^{T}(t-s) N F\left(x_{N}(s)\right) d s .
\end{aligned}
$$

Thus, comparing (4.8) and (4.2), we expect $x_{N}$ and $x$ to be close, for $0 \leq t \leq T<t_{\max }$, provided that we can show that $\sup _{t \leq T}\left\|\widetilde{m}_{N}(t)\right\|_{\mu}$ is small, where $\widetilde{m}_{N}(t):=N^{-1} \widetilde{M}_{N}(t)$. Indeed, if $x_{N}(0)$ and $x(0)$ are close, then

$$
\begin{aligned}
\| x_{N}(t)- & x(t) \|_{\mu} \\
\leq \| & R^{T}(t)\left(x_{N}(0)-x(0)\right) \|_{\mu} \\
& \quad+\int_{0}^{t}\left\|R^{T}(t-s)\left[F\left(x_{N}(s)\right)-F(x(s))\right]\right\|_{\mu} d s+\left\|\widetilde{m}_{N}(t)\right\|_{\mu} \\
\leq \quad & e^{w t}\left\|x_{N}(0)-x(0)\right\|_{\mu} \\
& \quad+\int_{0}^{t} e^{w(t-s)} K\left(\mu, F ; 2 \Xi_{T}\right)\left\|x_{N}(s)-x(s)\right\|_{\mu} d s+\left\|\widetilde{m}_{N}(t)\right\|_{\mu},
\end{aligned}
$$

by (3.12), with the stage apparently set for Gronwall's inequality, assuming that $\left\|x_{N}(0)-x(0)\right\|_{\mu}$ and $\sup _{0 \leq t \leq T}\left\|\widetilde{m}_{N}(t)\right\|_{\mu}$ are small enough that then $\left\|x_{N}(t)\right\|_{\mu} \leq 2 \Xi_{T}$ for $0 \leq t \leq T$, where $\Xi_{T}:=\sup _{0 \leq t \leq T}\|x(t)\|_{\mu}$.

Bounding $\sup _{0 \leq t \leq T}\left\|\widetilde{m}_{N}(t)\right\|_{\mu}$ is, however, not so easy. Since $\widetilde{M}_{N}$ is not itself a martingale, we cannot directly apply martingale inequalities to control its fluctuations. However, since

$$
\widetilde{M}_{N}(t)=\int_{0}^{t} R^{T}(t-s) d M_{N}(s)
$$

we can hope to use control over the martingale $M_{N}$ instead. For this and the subsequent argument, we introduce some further assumptions. 


\section{Assumption 4.2}

1. There exists $r=r_{\mu} \leq r_{\max }^{(2)}$ such that $\sup _{j \geq 0}\left\{\mu(j) / \nu_{r}(j)\right\}<\infty$.

2. There exists $\zeta \in \mathcal{R}$ with $\zeta(j) \geq 1$ for all $j$ such that (2.27) is satisfied for some $b=b(\zeta) \geq 1$ and $r=r(\zeta)$ such that $1 \leq r(\zeta) \leq r_{\max }^{(2)}$, and that

$$
Z:=\sum_{k \geq 0} \frac{\mu(k)\left(\left|A_{k k}\right|+1\right)}{\sqrt{\zeta(k)}}<\infty .
$$

Note that part 1 of Assumption 4.2 implies $\lim _{j \rightarrow \infty}\left\{\mu(j) / \nu_{r}(j)\right\}=0$ for some $r=\tilde{r}_{\mu} \leq r_{\mu}+1$. We define

$$
\rho(\zeta, \mu):=\max \left\{r(\zeta), p(r(\zeta)), \tilde{r}_{\mu}\right\}
$$

where $p(\cdot)$ is as in Assumptions 2.1. We can now prove the following lemma, which enables us to control the paths of $\widetilde{M}_{N}$ by using fluctuation bounds for the martingale $M_{N}$.

Lemma 4.3 Under Assumption 4.2,

$$
\widetilde{M}_{N}(t)=M_{N}(t)+\int_{0}^{t} R^{T}(t-s) A M_{N}(s) d s .
$$

Proof. Since $A$ generates $R^{T}$, it follows that

$$
R^{T}(t-s)=I+\int_{0}^{t-s} R^{T}(v) A d v .
$$

Substituting this into (4.11), we obtain

$$
\begin{aligned}
\widetilde{M}_{N}(t) & =\int_{0}^{t} R^{T}(\tau-s) d M_{N}(s) \\
& =M_{N}(t)+\int_{0}^{t}\left\{\int_{0}^{t} R^{T}(v) A 1_{\{v \leq t-s\}} d v\right\} d M_{N}(s) \\
& =M_{N}(t)+\int_{0}^{t}\left\{\int_{0}^{t} R^{T}(v) A 1_{\{v \leq t-s\}} d v\right\} d X_{N}(s) \\
& \quad-\int_{0}^{t}\left\{\int_{0}^{t} R^{T}(v) A 1_{\{v \leq t-s\}} d v\right\} F_{0}\left(x_{N}(s)\right) d s .
\end{aligned}
$$


It remains to change the order of integration in the double integrals, for which we use Fubini's theorem.

In the first, the outer integral is almost surely a finite sum, and at each jump time $t_{l}^{X_{N}}$ we have $d X_{N}\left(t_{l}^{X_{N}}\right) \in \mathcal{J}$. Hence it is enough that, for each $i$ and $m, \sum_{j \geq 0} R_{i j}^{T} A_{j m}$ is absolutely summable, which is immediate because there is at most one negative term in the sum. Thus we have

$$
\int_{0}^{t}\left\{\int_{0}^{t} R^{T}(v) A \mathbf{1}_{\{v \leq t-s\}} d v\right\} d X_{N}(s)=\int_{0}^{t} R^{T}(v) A\left\{X_{N}(t-v)-X_{N}(0)\right\} d v .
$$

For the second, the $k$-th component of $R^{T}(v) A F_{0}\left(x_{N}(s)\right)$ is just

$$
\sum_{j \geq 0} R_{j k}(v) \sum_{l \geq 0} A_{j l} \sum_{J \in \mathcal{J}} J^{l} \alpha^{J}\left(x_{N}(s)\right) .
$$

Now, from (3.4), we have $0 \leq R_{j k}(v) \leq \mu(j) e^{w v} / \mu(k)$, and

$$
\sum_{j \geq 0} \mu(j)\left|A_{j l}\right| \leq \mu(l)\left(2\left|A_{l l}\right|+w\right)
$$

because $A^{T} \mu \leq w \mu$. Hence, putting absolute values in the summands in (4.15) yields at most

$$
\frac{e^{w v}}{\mu(k)} \sum_{J \in \mathcal{J}} \alpha^{J}\left(x_{N}(s)\right) \sum_{l \geq 0}\left|J^{l}\right| \mu(l)\left(2\left|A_{l l}\right|+w\right) .
$$

Now, in view of (4.12) and since $\zeta(j) \geq 1$ for all $j$, there is a constant $K<\infty$ such that $\mu(l)\left(2\left|A_{l l}\right|+w\right) \leq K \zeta(l)$. Furthermore, $\zeta$ satisfies (2.27), so that, by Corollary $2.5, \sum_{J \in \mathcal{J}} \alpha^{J}\left(x_{N}(s)\right) \sum_{l \geq 0}\left|J^{l}\right| \zeta(l)$ is a.s. uniformly bounded in $0 \leq s \leq T$. Hence we can apply Fubini's theorem, obtaining

$$
\int_{0}^{t}\left\{\int_{0}^{t} R^{T}(v) A 1_{\{v \leq t-s\}} d v\right\} F_{0}\left(x_{N}(s)\right) d s=\int_{0}^{t} R^{T}(v) A\left\{\int_{0}^{t-v} F_{0}\left(x_{N}(s)\right) d s\right\} d v
$$

and combining this with (4.14) proves the lemma.

We now introduce the exponential martingales that we use to bound the fluctuations of $M_{N}$. For $\theta \in R^{\mathbb{Z}_{+}}$bounded and $x \in \mathcal{R}_{\mu}$,

$$
Z_{N, \theta}(t):=e^{\theta^{T} x_{N}(t)} \exp \left\{-\int_{0}^{t} g_{N \theta}\left(x_{N}(s-)\right) d s\right\}, \quad t \geq 0
$$


is a non-negative finite variation local martingale, where

$$
g_{N \theta}(\xi):=\sum_{J \in \mathcal{J}} N \alpha_{J}(\xi)\left(e^{N^{-1} J^{T} \theta}-1\right) .
$$

For $t \geq 0$, we have

$$
\begin{aligned}
\log Z_{N, \theta}(t) & =\theta^{T} x_{N}(t)-\int_{0}^{t} g_{N \theta}\left(x_{N}(s-)\right) d s \\
& =\theta^{T} m_{N}(t)-\int_{0}^{t} \varphi_{N, \theta}\left(x_{N}(s-), s\right) d s
\end{aligned}
$$

where

$$
\varphi_{N, \theta}(\xi):=\sum_{J \in \mathcal{J}} N \alpha_{J}(\xi)\left(e^{N^{-1} J^{T} \theta}-1-N^{-1} J^{T} \theta\right),
$$

and $m_{N}(t):=N^{-1} M_{N}(t)$. Note also that we can write

$$
\varphi_{N, \theta}(\xi)=N \int_{0}^{1}(1-r) D^{2} m_{N}(\xi, r \theta)[\theta, \theta] d r
$$

where

$$
m_{N}\left(\xi, \theta^{\prime}\right):=\sum_{J \in \mathcal{J}} \alpha_{J}(\xi) e^{N^{-1} J^{T} \theta^{\prime}}
$$

and $D^{2} m$ denotes its matrix of second derivatives with respect to $\theta^{\prime}$ :

$$
D^{2} m\left(\xi, \theta^{\prime}\right)\left[\zeta_{1}, \zeta_{2}\right]:=N^{-2} \sum_{J \in \mathcal{J}} \alpha_{J}(\xi) e^{N^{-1} J^{T} \theta^{\prime}} \zeta_{1}^{T} J J^{T} \zeta_{2}
$$

for any $\zeta_{1}, \zeta_{2} \in \mathcal{R}_{\mu}$.

Now choose any $B:=\left(B_{k}, k \geq 0\right) \in \mathcal{R}$, and define $\tilde{\tau}_{k}^{(N)}(B)$ by

$$
\tilde{\tau}_{k}^{(N)}(B):=\inf \left\{t \geq 0: \sum_{J: J_{k} \neq 0} \alpha_{J}\left(x_{N}(t-)\right)>B_{k}\right\} .
$$

Our exponential bound is as follows.

Lemma 4.4 For any $k \geq 0$,

$$
\mathbf{P}\left[\sup _{0 \leq t \leq T \wedge \tilde{\tau}_{k}^{(N)}(B)}\left|m_{N}^{k}(t)\right| \geq \delta\right] \leq 2 \exp \left(-\delta^{2} N / 2 B_{k} K_{*} T\right)
$$

for all $0<\delta \leq B_{k} K_{*} T$, where $K_{*}:=J_{*}^{2} e^{J_{*}}$, and $J_{*}$ is as in (1.1). 
Proof. Take $\theta=e^{(k)} \beta$, for $\beta$ to be chosen later, Apply the optional stopping theorem to the martingale $Z_{N, \theta}$ with stopping time $\sigma^{(N)}(k, \delta)$, where

$$
\sigma^{(N)}(k, \delta):=T \wedge \tilde{\tau}_{k}^{(N)}(B) \wedge \inf \left\{t: m_{N}^{k}(t) \geq \delta\right\}
$$

Note that

$$
e^{N^{-1} J^{T} \theta} \leq e^{J_{*}}
$$

so long as $|\beta| \leq N$. Hence

$$
D^{2} m(\xi, r \theta)[\theta, \theta] \leq N^{-2}\left(\sum_{J: J_{k} \neq 0} \alpha_{J}(\xi)\right) \beta^{2} K_{*}
$$

Thus, from (4.19), we have

$$
\varphi_{N, \theta}\left(x_{N}(u-)\right) \leq \frac{1}{2} N^{-1} B_{k} \beta^{2} K_{*}, \quad u \leq \tilde{\tau}_{k}^{(N)}(B)
$$

On the event that $\sigma^{(N)}(k, \delta)=\inf \left\{t: m_{N}^{k}(t) \geq \delta\right\} \leq\left(T \wedge \tilde{\tau}_{k}^{(N)}(B)\right)$,

$$
Z_{N, \theta}(\sigma(k, \delta)) \geq \exp \left\{\beta \delta-\frac{1}{2} N^{-1} B_{k} \beta^{2} K_{*} T\right\}
$$

But since $Z_{N, \theta}(0)=1$, it now follows that

$$
\begin{aligned}
1 & \geq \mathbf{E}\left\{Z_{N, \theta}(\sigma(k, \delta))\right\} \\
& \geq \mathbf{P}\left[\sup _{0 \leq t \leq T \wedge \tilde{\tau}_{k}^{(N)}(B)} m_{N}^{k}(t) \geq \delta\right] \exp \left\{\beta \delta-\frac{1}{2} N^{-1} B_{k} \beta^{2} K_{*} T\right\}
\end{aligned}
$$

We can choose $\beta=\delta N / B_{k} K_{*} T$, as long as $\delta / B_{k} K_{*} T \leq 1$, obtaining

$$
\mathbf{P}\left(\sup _{0 \leq t \leq T \wedge \tilde{\tau}_{k}^{(N)}(B)} m_{N}^{k}(t) \geq \delta\right) \leq \exp \left(-\delta^{2} N / 2 B_{k} K_{*} T\right)
$$

Repeating with

$$
\tilde{\sigma}^{(N)}(k, \delta):=T \wedge \tilde{\tau}_{k}^{(N)}(B) \wedge \inf \left\{t:-m_{N}^{k}(t) \geq \delta\right\},
$$

and choosing $\beta=\delta N / B_{k} K_{*} T$, gives the lemma. 
The preceding lemma gives a bound for each individual component of $M_{N}$. We need first to translate this into a statement for all components simultaneously. For $\zeta$ as in Assumption 4.2, we start by writing

$$
Z_{*}^{(1)}:=\max _{k \geq 1} k^{-1} \#\{m: \zeta(m) \leq k\} ; \quad Z_{*}^{(2)}:=\sup _{k \geq 0} \frac{\mu(k)\left(\left|A_{k k}\right|+1\right)}{\sqrt{\zeta(k)}}
$$

both finite because of Assumption 4.2. Then, using the definition (2.26) of $\tau^{(N)}(a, \zeta)$, note that

$$
\sum_{J: J^{k} \neq 0} \alpha\left(x_{N}(t)\right) h(k) \leq \sum_{J: J^{k} \neq 0} \frac{\alpha\left(x_{N}(t)\right) h(k) d(J, \zeta)}{\left|J^{k}\right| \zeta(k)} \leq \frac{a h(k)}{\zeta(k)}
$$

for any $t \leq \tau^{(N)}(a, \zeta)$ and any $h \in \mathcal{R}$, and that, for any $\mathcal{K} \subseteq \mathbb{Z}_{+}$,

$$
\begin{aligned}
\sum_{k \in \mathcal{K}} \sum_{J: J^{k} \neq 0} \alpha\left(x_{N}(t)\right) h(k) & \leq \sum_{k \in \mathcal{K}} \sum_{J: J^{k} \neq 0} \frac{\alpha\left(x_{N}(t)\right) h(k) d(J, \zeta)}{\left|J^{k}\right| \zeta(k)} \\
& \leq \frac{a}{\min _{k \in \mathcal{K}}(\zeta(k) / h(k))}
\end{aligned}
$$

From (4.23) with $h(k)=1$ for all $k$, if we choose $B:=(a / \zeta(k), k \geq 0)$, then $\tau^{(N)}(a, \zeta) \leq \tilde{\tau}_{k}^{(N)}(B)$ for all $k$. For this choice of $B$, we can take

$$
\delta_{k}^{2}:=\delta_{k}^{2}(a):=\frac{4 a K_{*} T \log N}{N \zeta(k)}
$$

in Lemma 4.4 for $k \in \kappa_{N}(a)$, where

$$
\kappa_{N}(a):=\left\{k: \zeta(k) \leq \frac{1}{4} a K_{*} T N / \log N\right\},
$$

since then $\delta_{k}(a) \leq B_{k} K_{*} T$. Note that then, from (4.12),

$$
\sum_{k \in \kappa_{N}(a)} \mu(k) \delta_{k}(a) \leq 2 Z \sqrt{a K_{*} T} \sqrt{\frac{\log N}{N}}
$$

with $Z$ as defined in Assumption 4.2, and that

$$
\left|\kappa_{N}(a)\right| \leq \frac{1}{4} a Z_{*}^{(1)} K_{*} T N / \log N
$$


Lemma 4.5 If Assumptions 4.2 are satisfied, taking $\delta_{k}(a)$ and $\kappa_{N}(a)$ as defined in (4.25) and (4.26), and for any $\eta \in \mathcal{R}$, we have
1. $\quad \mathbf{P}\left[\bigcup_{k \in \kappa_{N}(a)}\left\{\sup _{0 \leq t \leq T \wedge \tau^{(N)}(a, \zeta)}\left|m_{N}(t)\right| \geq \delta_{k}(a)\right\}\right] \leq \frac{a Z_{*}^{(1)} K_{*} T}{2 N \log N}$
2. $\quad \mathbf{P}\left[\sum_{k \notin \kappa_{N}(a)} X_{N}^{k}(t)=0\right.$ for all $\left.0 \leq t \leq T \wedge \tau^{(N)}(a, \zeta)\right] \geq 1-\frac{4 \log N}{K_{*} N}$;
3. $\sup _{0 \leq t \leq T \wedge \tau^{(N)}(a, \zeta)}\left\{\sum_{k \notin \kappa_{N}(a)} \eta(k)\left|F^{k}\left(x_{N}(t)\right)\right|\right\} \leq \frac{a J_{*}}{\min _{k \notin \kappa_{N}(a)}(\zeta(k) / \eta(k))}$.

Proof. For part 1, use Lemma 4.4 together with (4.25) and (4.28) to give the bound. For part 2, the total rate of jumps into coordinates with indices $k \notin \kappa_{N}(a)$ is

$$
\sum_{k \notin \kappa_{N}(a)} \sum_{J: J^{k} \neq 0} \alpha\left(x_{N}(t)\right) \leq \frac{a}{\min _{k \notin \kappa_{N}(a)} \zeta(k)},
$$

if $t \leq \tau^{(N)}(a, \zeta)$, using (4.24) with $\mathcal{K}=\left(\kappa_{N}(a)\right)^{c}$, which, combined with (4.26), proves the claim. For the final part, if $t \leq \tau^{(N)}(a, \zeta)$,

$$
\sum_{k \notin \kappa_{N}(a)} \eta(k)\left|F^{k}\left(x_{N}(t)\right)\right| \leq \sum_{k \notin \kappa_{N}(a)} \eta(k) \sum_{J: J^{k} \neq 0} \alpha\left(x_{N}(t)\right) J_{*},
$$

and the inequality follows once more from (4.24).

Let $B_{N}^{(1)}(a)$ and $B_{N}^{(2)}(a)$ denote the events

$$
\begin{aligned}
B_{N}^{(1)}(a) & :=\left\{\sum_{k \notin \kappa_{N}(a)} X_{N}^{k}(t)=0 \text { for all } 0 \leq t \leq T \wedge \tau^{(N)}(a, \zeta)\right\} \\
B_{N}^{(2)}(a) & :=\left(\bigcap_{k \in \kappa_{N}(a)}\left\{\sup _{0 \leq t \leq T \wedge \tau^{(N)}(a, \zeta)} \mid m_{N}(\text { inTheorem } 2.4 t) \mid \leq \delta_{k}(a)\right\}(4) 29\right)
\end{aligned}
$$

and set $B_{N}(a):=B_{N}^{(1)}(a) \cap B_{N}^{(2)}(a)$. Then, by Lemma 4.5 , we deduce that

$$
\mathbf{P}\left[B_{N}(a)^{c}\right] \leq \frac{a Z_{*}^{(1)} K_{*} T}{2 N \log N}+\frac{4 \log N}{K_{*} N},
$$


of order $O\left(N^{-1} \log N\right)$ for each fixed $a$. Thus we have all the components of $M_{N}$ simultaneously controlled, except on a set of small probability. We now translate this into the desired assertion about the fluctuations of $\widetilde{m}_{N}$.

Lemma 4.6 If Assumptions 4.2 are satisfied, then, on the event $B_{N}(a)$,

$$
\sup _{0 \leq t \leq T \wedge \tau^{(N)}(a, \zeta)}\left\|\widetilde{m}_{N}(t)\right\|_{\mu} \leq \sqrt{a} K_{4.6} \sqrt{\frac{\log N}{N}}
$$

where the constant $K_{4.6}$ depends on $T$ and the parameters of the process.

Proof. From Lemma 4.3, it follows that

$$
\begin{aligned}
& \sup _{0 \leq t \leq T \wedge \tau^{(N)}(a, \zeta)}\left\|\tilde{m}_{N}(t)\right\|_{\mu} \\
& \quad \leq \sup _{0 \leq t \leq T \wedge \tau^{(N)}(a, \zeta)}\left\|m_{N}(t)\right\|_{\mu}+\sup _{0 \leq t \leq T \wedge \tau^{(N)}(a, \zeta)} \int_{0}^{t}\left\|R^{T}(t-s) A m_{N}(s)\right\|_{\mu} d s .
\end{aligned}
$$

For the first term, on $B_{N}(a)$ and for $0 \leq t \leq T \wedge \tau^{(N)}(a, \zeta)$, we have

$$
\left\|m_{N}(t)\right\|_{\mu} \leq \sum_{k \in \kappa_{N}(a)} \mu(k) \delta_{k}(a)+\int_{0}^{t} \sum_{k \notin \kappa_{N}(a)} \mu(k)\left|F^{k}\left(x_{N}(u)\right)\right| d u .
$$

The first sum is bounded using $(4.27)$ by $2 Z \sqrt{a K_{*} T} N^{-1 / 2} \sqrt{\log N}$, the second, from Lemma 4.5 and (4.26), by

$$
\frac{T a J_{*}}{\min _{k \notin \kappa_{N}(a)}(\zeta(k) / \mu(k))} \leq Z_{*}^{(2)} 2 J_{*} \sqrt{\frac{T a}{K_{*}}} \sqrt{\frac{\log N}{N}}
$$

For the second term in (4.31), from (3.4) and (4.16), we note that

$$
\begin{aligned}
\left\|R^{T}(t-s) A m_{N}(s)\right\|_{\mu} & \leq \sum_{k \geq 0} \mu(k) \sum_{l \geq 0} R_{l k}(t-s) \sum_{r \geq 0}\left|A_{l r}\right|\left|m_{N}^{r}(s)\right| \\
& \leq e^{w(t-s)} \sum_{l \geq 0} \mu(l) \sum_{r \geq 0}\left|A_{l r}\right|\left|m_{N}^{r}(s)\right| \\
& \leq e^{w(t-s)} \sum_{r \geq 0} \mu(r)\left\{2\left|A_{r r}\right|+w\right\}\left|m_{N}^{r}(s)\right|
\end{aligned}
$$


On $B_{N}(a)$ and for $0 \leq s \leq T \wedge \tau^{(N)}(a, \zeta)$, from (4.12), the sum for $r \in \kappa_{N}(a)$ is bounded using

$$
\begin{aligned}
& \sum_{r \in \kappa_{N}(a)} \mu(r)\left\{2\left|A_{r r}\right|+w\right\}\left|m_{N}^{r}(s)\right| \\
& \leq \sum_{r \in \kappa_{N}(a)} \mu(r)\left\{2\left|A_{r r}\right|+w\right\} \delta_{r}(a) \\
& \leq \sum_{r \in \kappa_{N}(a)} \mu(r)\left\{2\left|A_{r r}\right|+w\right\} \sqrt{\frac{4 a K_{*} T \log N}{N \zeta(r)}} \\
& \leq(2 \vee w) Z \sqrt{4 a K_{*} T} \sqrt{\frac{\log N}{N}} .
\end{aligned}
$$

The remaining sum is then bounded by Lemma 4.5 , on $B_{N}(a)$ and for $0 \leq$ $s \leq T \wedge \tau^{(N)}(a, \zeta)$ giving at most

$$
\begin{aligned}
& \sum_{r \notin \kappa_{N}(a)} \mu(r)\left\{2\left|A_{r r}\right|+w\right\}\left|m_{N}^{r}(s)\right| \\
& \leq \sum_{r \notin \kappa_{N}(a)} \mu(r)\left\{2\left|A_{r r}\right|+w\right\} \int_{0}^{s}\left|F^{r}\left(x_{N}(t)\right)\right| d t \\
& \leq \frac{(2 \vee w) s a J_{*}}{\min _{k \notin \kappa_{N}(a)}\left(\zeta(k) / \mu(k)\left\{\left|A_{k k}\right|+1\right\}\right)} \\
& \leq(2 \vee w) Z_{*}^{(2)} 2 J_{*} \sqrt{\frac{T a}{K_{*}}} \sqrt{\frac{\log N}{N}} .
\end{aligned}
$$

Integrating, it follows that

$$
\begin{aligned}
& \sup _{0 \leq t \leq T \wedge \tau^{(N)}(a, \zeta)} \int_{0}^{t}\left\|R^{T}(t-s) A m_{N}(s)\right\|_{\mu} d s \\
& \leq(2 T \vee 1) e^{w T}\left\{\sqrt{4 a K_{*} T} Z+Z_{*}^{(2)} J 2 J_{*} \sqrt{\frac{T a}{K_{*}}}\right\} \sqrt{\frac{\log N}{N}},
\end{aligned}
$$

and the lemma follows.

This has now established the control on $\sup _{0 \leq t \leq T}\left\|\widetilde{m}_{N}(t)\right\|_{\mu}$ that we need, in order to translate (4.10) into a proof of the main theorem. 
Theorem 4.7 Suppose that (1.1), (1.2), (3.1), (3.2) and (4.1) are all satisfied, and that Assumptions 2.1 and 4.2 hold. Recalling the definition (4.13) of $\rho(\zeta, \mu)$, for $\zeta$ as given in Assumption 4.2, suppose that $S_{\rho(\zeta, \mu)}^{(N)}(0) \leq N C_{*}$ for some $C_{*}<\infty$.

Let $x$ denote the solution to (4.2) with initial condition $x(0)$ satisfying $S_{\rho(\zeta, \mu)}(x(0))<\infty$. Then $t_{\max }=\infty$.

Fix any $T$, and define $\Xi_{T}:=\sup _{0 \leq t \leq T}\|x(t)\|_{\mu}$. If $\left\|x_{N}(0)-x(0)\right\|_{\mu} \leq$ $\frac{1}{2} \Xi_{T} e^{-\left(w+k_{*}\right) T}$, where $k_{*}:=e^{w T} K\left(\mu, F ; 2 \Xi_{T}\right)$, then there exist constants $c_{1}, c_{2}$ depending on $C_{*}, T$ and the parameters of the process, such that for all $N$ large enough

$\mathbf{P}\left(\sup _{0 \leq t \leq T}\left\|x_{N}(t)-x(t)\right\|_{\mu}>\left(e^{w T}\left\|x_{N}(0)-x(0)\right\|_{\mu}+c_{1} \sqrt{\frac{\log N}{N}}\right) e^{k_{*} T}\right) \leq \frac{c_{2} \log N}{N}$.

Proof. As $S_{\rho(\zeta, \mu)}^{(N)}(0) \leq N C_{*}$, it follows also that $S_{r}^{(N)}(0) \leq N C_{*}$ for all $0 \leq r \leq \rho(\zeta, \mu)$. Fix any $T<t_{\max }$, take $C:=2\left(C_{*}+k_{04} T\right) e^{k_{01} T}$, and observe that, for $r \leq \rho(\zeta, \mu) \wedge r_{\max }^{(2)}$, and such that $p(r) \leq \rho(\zeta, \mu)$, we can take

$$
C_{r T}^{\prime \prime} \leq \widetilde{C}_{r T}:=\left\{2\left(C_{*} \vee 1\right)+k_{r 4} T\right\} e^{\left(k_{r 1}+C k_{r 2}\right) T},
$$

in Theorem 2.4, since we can take $C_{*}$ to bound $C_{r}$ and $C_{r}^{\prime}$. In particular, by definition, the inequality is satisfied for $r=r(\zeta)$, as defined in Assumption 4.2. Then, taking $a:=\left\{k_{2}+k_{1} \widetilde{C}_{r(\zeta) T}\right\}^{b(\zeta)}$ in Corollary 2.5, it follows that for some constant $c_{3}>0$, on the event $B_{N}(a)$,

$$
\mathbf{P}\left[\tau^{(N)}(a, \zeta) \leq T\right] \leq c_{3} N^{-1} .
$$

Then, from (4.30), for some constant $c_{4}, \mathbf{P}\left[B_{N}(a)^{c}\right] \leq c_{4} N^{-1} \log N$. Here, constants $c_{3}, c_{4}$ depend on $C_{*}, T$ and the parameters of the process.

We now use Lemma 4.6 to bound the martingale term in (4.10). It follows that, on the event $B_{N}(a) \cap\left\{\tau^{(N)}(a, \zeta)>T\right\}$ and on the event that $\left\|x_{N}(s)-x(s)\right\|_{\mu} \leq \Xi_{T}$ for all $0 \leq s \leq t$,

$$
\begin{aligned}
& \left\|x_{N}(t)-x(t)\right\|_{\mu} \leq\left(e^{w T}\left\|x_{N}(0)-x(0)\right\|_{\mu}+\sqrt{a} K_{4.6} \sqrt{\frac{\log N}{N}}\right) \\
& +k_{*} \int_{0}^{t}\left\|x_{N}(s)-x(s)\right\|_{\mu} d s,
\end{aligned}
$$


where $k_{*}:=e^{w T} K\left(\mu, F ; 2 \Xi_{T}\right)$. Then from Gronwall's inequality, on the event $B_{N}(a) \cap\left\{\tau^{(N)}(a, \zeta)>T\right\}$,

$$
\left\|x_{N}(t)-x(t)\right\|_{\mu} \leq\left(e^{w T}\left\|x_{N}(0)-x(0)\right\|_{\mu}+\sqrt{a} K_{4.6} \sqrt{\frac{\log N}{N}}\right) e^{k^{*} t}
$$

for all $0 \leq t \leq T$, provided that

$$
\left(e^{w T}\left\|x_{N}(0)-x(0)\right\|_{\mu}+\sqrt{a} K_{4.6} \sqrt{\frac{\log N}{N}}\right) \leq \Xi_{T} e^{-k^{*} T} .
$$

This is true for all $N$ sufficiently large, if $\left\|x_{N}(0)-x(0)\right\|_{\mu} \leq \frac{1}{2} \Xi_{T} e^{-\left(w+k^{*}\right) T}$, which we have assumed. We have thus proved (4.32), since, as shown above, $\mathbf{P}\left(B_{N}(a)^{c} \cup\left\{\tau^{(N)}(a, \zeta)>T\right\}^{c}\right)=O\left(N^{-1} \log N\right)$.

We now use this to show that in fact $t_{\max }=\infty$. For $x(0)$ as above, we can take $x_{N}^{j}(0):=N^{-1}\left\lfloor N x^{j}(0)\right\rfloor \leq x^{j}(0)$, so that $S_{\rho(\zeta, \mu)}^{(N)}(0) \leq N C_{*}$ for $C_{*}:=$ $S_{\rho(\zeta, \mu)}(x(0))<\infty$. Then, by (4.13), $\lim _{j \rightarrow \infty}\left\{\mu(j) / \nu_{\rho(\zeta, \mu)}(j)\right\}=0$, so it follows easily using bounded convergence that $\left\|x_{N}(0)-x(0)\right\|_{\mu} \rightarrow 0$ as $N \rightarrow \infty$. Hence, for any $T<t_{\max }$, it follows from (4.32) that $\left\|x_{N}(t)-x(t)\right\|_{\mu} \rightarrow_{D} 0$ as $N \rightarrow \infty$, for $t \leq T$, with uniform bounds over the interval, where ' $\rightarrow{ }_{D}$ ' denotes convergence in distribution. Also, by Assumption 4.2, there is a constant $c_{5}$ such that $\left\|x_{N}(t)\right\|_{\mu} \leq c_{5} N^{-1} S_{r_{\mu}}^{(N)}(t)$ for each $t$, where $r_{\mu} \leq r_{\max }^{(2)}$ and $r_{\mu} \leq \rho(\zeta, \mu)$. Hence, using Lemma 2.3 and Theorem 2.4, $\sup _{0 \leq t \leq 2 T}\left\|x_{N}(t)\right\|_{\mu}$ remains bounded in probability as $N \rightarrow \infty$. Hence it is impossible that $\|x(t)\|_{\mu} \rightarrow \infty$ as $T \rightarrow t_{\max }<\infty$, implying that in fact $t_{\max }=\infty$ for such $x(0)$.

Remark. The dependence on the initial conditions is considerably complicated by the way the constant $C$ appears in the exponent, for instance in the expression for $\widetilde{C}_{r T}$ in the proof of Theorem 4.7. However, if $k_{r 2}$ in Assumptions 2.1 can be chosen to be zero, as for instance in the examples below, the dependence simplifies correspondingly.

\section{$5 \quad$ Examples}

We begin with some general remarks, to show that the assumptions are satisfied in many practical contexts. We then discuss two particular examples, 
those of Kretzschmar (1993) and of Arrigoni (2003), that fitted poorly or not at all into the general setting of Barbour \& Luczak (2008), though the other systems referred to in the introduction could also be treated similarly. In both of our chosen examples, the index $j$ represents a number of individuals - parasites in a host in the first, animals in a patch in the second and we shall for now use the former terminology for the preliminary, general discussion.

Transitions that can typically be envisaged are: births of a few parasites, which may occur either in the same host, or in another, if infection is being represented; births and immigration of hosts, with or without parasites; migration of parasites between hosts; deaths of parasites; deaths of hosts; and treatment of hosts, leading to the deaths of many of the host's parasites. For births of parasites, there is a transition $X \rightarrow X+J$, where $J$ takes the form

$$
J_{l}=1 ; \quad J_{m}=-1 ; \quad J_{j}=0, j \neq l, m,
$$

indicating that one $m$-host has become an $l$-host. For births of parasites within a host, a transition rate of the form $b_{l-m} m X_{m}$ could be envisaged, with $l>m$, the interpretation being that there are $X_{m}$ hosts with parasite burden $m$, each of which gives birth to $s$ offspring at rate $b_{s}$, for some small values of $s$. For infection of an $m$-host, a possible transition rate would be of the form

$$
X_{m} \sum_{j \geq 0} N^{-1} X_{j} \lambda p_{j, l-m},
$$

since an $m$-host comes into contact with $j$-hosts at a rate proportional to their density in the host population, and $p_{j r}$ represents the probability of a $j$-host transferring $r$ parasites to the infected host during the contact. The probability distributions $p_{j}$. can be expected to be stochastically increasing in $j$. Deaths of parasites also give rise to transitions of the form (5.1), but now with $l<m$, the simplest form of rate being just $d m X_{m}$ for $l=$ $m-1$, though $d=d_{m}$ could also be chosen to increase with parasite burden. Treatment of a host would lead to values of $l$ much smaller than $m$, and a rate of the form $\kappa X_{m}$ for the transition with $l=0$ would represent fully successful treatment of randomly chosen individuals. Births and deaths of hosts and immigration all lead to transitions of the form

$$
J_{l}= \pm 1 ; \quad J_{j}=0, j \neq l .
$$

For deaths, $J_{l}=-1$, and a typical rate would be $d^{\prime} X_{l}$. For births, $J_{l}=1$, and a possible rate would be $\sum_{j \geq 0} X_{j} b_{j l}^{\prime}$ (with $l=0$ only, if new-born individuals 
are free of parasites). For immigration, constant rates $\lambda_{l}$ could be supposed. Finally, for migration of individual parasites between hosts, transitions are of the form

$J_{l}=J_{m}=-1 ; \quad J_{l+1}=1 ; \quad J_{m-1}=1 ; \quad J_{j}=0, j \neq l, m, l+1, m-1$,

a possible rate being $\gamma m X_{m} N^{-1} X_{l}$.

For all the above transitions, we can take $J_{*}=2$ in (1.1), and (1.2) is satisfied in biologically sensible models. (3.1) and (3.2) depend on the way in which the matrix $A$ can be defined, which is more model specific; in practice, (3.1) is very simple to check. The choice of $\mu$ in (3.2) is influenced by the need to have (4.1) satisfied. For Assumptions 2.1, a possible choice of $\nu$ is to take $\nu(j)=(j+1)$ for each $j \geq 0$, with $S_{1}(X)$ then representing the number of hosts plus the number of parasites. Satisfying (2.5) is then easy for transitions only involving the movement of a single parasite, but in general requires assumptions as to the existence of the $r$-th moments of the distributions of the numbers of parasites introduced at birth, immigration and infection events. For (2.6), in which transitions involving a net reduction in the total number of parasites and hosts can be disregarded, the parasite birth events are those in which the rates typically have a factor $m X_{m}$ for transitions with $J_{m}=-1$, with $m$ in principle unbounded. However, at such events, an $m$-individual changes to an $m+s$ individual, with the number $s$ of offspring of the parasite being typically small, so that the value of $J^{T} \nu_{r}$ associated with this rate has magnitude $m^{r-1}$; the product $m X_{m} m^{r-1}$, when summed over $m$, then yields a contribution of magnitude $S_{r}(X)$, which is allowable in (2.6). Similar considerations show that the terms $N^{-1} S_{0}(X) S_{r}(X)$ accommodate the migration rates suggested above. Finally, in order to have Assumptions 4.2 satisfied, it is in practice necessary that Assumptions 2.1 are satisfied for large values of $r$, thereby imposing restrictions on the distributions of the numbers of parasites introduced at birth, immigration and infection events, as above. 


\subsection{Kretzschmar's model}

Kretzschmar (1993) introduced a model of a parasitic infection, in which the transitions from state $X$ are as follows:

$$
\begin{aligned}
& J=e^{(i-1)}-e^{(i)} \quad \text { at rate } \quad N i \mu x^{i}, \quad i \geq 1 ; \\
& J=-e^{(i)} \quad \text { at rate } N(\kappa+i \alpha) x^{i}, \quad i \geq 0 \text {; } \\
& J=e^{(0)} \quad \text { at rate } N \beta \sum_{i \geq 0} x^{i} \theta^{i} \\
& J=e^{(i+1)}-e^{(i)} \text { at rate } N \lambda x^{i} \varphi(x), \quad i \geq 0,
\end{aligned}
$$

where $x:=N^{-1} X, \varphi(x):=\|x\|_{11}\left\{c+\|x\|_{1}\right\}^{-1}$ with $c>0$, and $\|x\|_{11}:=$ $\sum_{j \geq 1} j|x|^{j}$; here, $0 \leq \theta \leq 1$, and $\theta^{i}$ denotes its $i$-th power (our $\theta$ corresponds to constant $\xi$ in [6]). Both (1.1) and (1.2) are obviously satisfied. For Assumptions (3.1), (3.2) and (4.1), we note that equation corresponding to (1.4) has

$$
\begin{aligned}
& A_{i i}=-\{\kappa+i(\alpha+\mu)\} ; \quad A_{i, i-1}^{T}=i \mu \text { and } A_{i 0}^{T}=\beta \theta^{i}, \quad i \geq 2 \\
& A_{11}=-\{\kappa+\alpha+\mu\} ; \quad A_{10}^{T}=\mu+\beta \theta \\
& A_{00}=-\kappa+\beta, \quad i \geq 1
\end{aligned}
$$

with all other elements of the matrix equal to zero, and

$$
F^{i}(x)=\lambda\left(x^{i-1}-x^{i}\right) \varphi(x), \quad i \geq 1 ; \quad F^{0}(x)=-\lambda x^{0} \varphi(x) .
$$

Hence Assumption (3.1) is immediate, and Assumption (3.2) holds for $\mu(j)=$ $(j+1)^{s}$, for any $s \geq 0$, with $w=(\beta-\kappa)_{+}$. The choice $\mu(j)=j+1$ then makes $F$ map elements of $\mathcal{R}_{\mu}$ to $\mathcal{R}_{\mu}$, and also locally Lipschitz in the $\mu$-norm, with $K(\mu, F ; \Xi)=c^{-2} \lambda \Xi(2 c+\Xi)$.

For Assumptions 2.1, choose $\nu=\mu$; then (2.5) is a finite sum for each $r \geq 0$. Turning to (2.6), it is immediate that $N U_{0 N}(X) \leq \beta S_{0}(X)$. Then

$$
\begin{aligned}
\sum_{i \geq 0} \lambda \varphi\left(N^{-1} X\right) X^{i}\left\{(i+2)^{r}-(i+1)^{r}\right\} & \leq \lambda \frac{S_{1}(X)}{S_{0}(X)} \sum_{i \geq 0} r X^{i}(i+2)^{r-1} \\
& \leq r 2^{r-1} \lambda S_{r}(X)
\end{aligned}
$$

since, by Jensen's inequality, $S_{1}(X) S_{r-1}(X) \leq S_{0}(X) S_{r}(X)$, and we can take $k_{r 2}=k_{r 4}=0$ and $k_{r 1}=\beta+r 2^{r-1} \lambda$ in $(2.6)$, for any $r \geq 1$, so $r_{\max }^{(1)}=\infty$. Finally, for (2.7),

$$
N V_{0 N}(X) \leq(\kappa+\beta) S_{0}(X)+\alpha S_{1}(X)
$$


so that $k_{03}=\kappa+\beta+\alpha$ and $k_{05}=0$, and

$$
\begin{aligned}
& N V_{r N}(X) \\
& \quad \leq r^{2}\left(\kappa S_{2 r}(X)+\alpha S_{2 r+1}(X)+\mu S_{2 r-1}(X)+2^{2(r-1)} \lambda S_{2 r-1}(X)\right)+\beta S_{0}(X),
\end{aligned}
$$

so that we can take $p(r)=2 r+1, k_{r 3}=\beta+r^{2}\left\{\kappa+\alpha+\mu+2^{2(r-1)} \lambda\right\}$, and $k_{r 5}=0$ for any $r \geq 1$, and so $r_{\max }^{(2)}=\infty$. In Assumptions 4.2, we can clearly take $r_{\mu}=1$ and $\zeta(k)=(k+1)^{7}$, giving $r(\zeta)=8, b(\zeta)=1$ and $\rho(\zeta, \mu)=17$.

\subsection{Arrigoni's model}

In the metapopulation model of Arrigoni (2003), the transitions from state $X$ are as follows:

$$
\begin{aligned}
& J=e^{(i-1)}-e^{(i)} \\
& \text { at rate } N i x^{i}\left(d_{i}+\gamma(1-\rho)\right), \quad i \geq 2 \text {; } \\
& J=e^{(0)}-e^{(1)} \\
& \text { at rate } N x^{1}\left(d_{1}+\gamma(1-\rho)+\kappa\right) \text {; } \\
& J=e^{(i+1)}-e^{(i)} \\
& \text { at rate } N i b_{i} x^{i} \\
& i \geq 1 \\
& J=e^{(0)}-e^{(i)} \\
& \text { at rate } N x^{i} \kappa \\
& i \geq 2 \text {; } \\
& J=e^{(k+1)}-e^{(k)}+e^{(i-1)}-e^{(i)} \text { at rate } N i x^{i} x^{k} \rho \gamma, \\
& k \geq 0, \quad i \geq 1 \text {. }
\end{aligned}
$$

Here, the total number $N$ of patches remains constant throughout, and the number of animals in any one patch changes by at most one at each transition; in the final (migration) transition, however, the numbers in two patches change simultaneously. In the above transitions, $\gamma, \rho, \kappa$ are non-negative, and $\left(d_{i}\right),\left(b_{i}\right)$ are sequences of non-negative numbers.

Once again, both (1.1) and (1.2) are obviously satisfied. The equation corresponding to (1.3) can now be expressed by taking

$$
\begin{aligned}
& A_{i i}=-\left\{\kappa+i\left(b_{i}+d_{i}+\gamma\right)\right\} ; \quad A_{i, i-1}^{T}=i\left(d_{i}+\gamma\right) ; \quad A_{i, i+1}^{T}=i b_{i}, \quad i \geq 1 ; \\
& A_{00}=-\kappa
\end{aligned}
$$

with all other elements of $A$ equal to zero, and

$$
F^{i}(x)=\rho \gamma\|x\|_{11}\left(x^{i-1}-x^{i}\right), \quad i \geq 1 ; \quad F^{0}(x)=-\rho \gamma x^{0}\|x\|_{11}+\kappa,
$$

where we have used the fact that $N^{-1} \sum_{j \geq 0} X_{j}=1$. Hence Assumption (3.1) is again immediate, and Assumption (3.2) holds for $\mu(j)=1$ with $w=0$, for $\mu(j)=j+1$ with $w=\max _{i}\left(b_{i}-d_{i}-\gamma-\kappa\right)_{+}\left(\operatorname{assuming}\left(b_{i}\right)\right.$ and 
$\left(d_{i}\right)$ to be such that this is finite), or indeed for $\mu(j)=(j+1)^{s}$ with any $s \geq 2$, with appropriate choice of $w$. The choice $\mu(j)=j+1$ again makes $F$ map elements of $\mathcal{R}_{\mu}$ to $\mathcal{R}_{\mu}$, and also locally Lipschitz in the $\mu$-norm, with $K(\mu, F ; \Xi)=3 \rho \gamma \Xi$.

To check Assumptions 2.1, take $\nu=\mu$; once again, (2.5) is a finite sum for each $r$. Then, for (2.6), it is immediate that $N U_{0 N}(X)=0$. For any $r \geq 1$, using arguments from the previous example,

$$
\begin{aligned}
N U_{r N}(X) & \leq r 2^{r-1}\left\{\sum_{i \geq 1} i b_{i} X^{i}(i+1)^{r-1}+\sum_{i \geq 1} \sum_{k \geq 0} i \rho \gamma N^{-1} X^{i} X^{k}(k+1)^{r-1}\right\} \\
& \leq r 2^{r-1}\left\{\max _{i} b_{i} S_{r}(X)+\rho \gamma N^{-1} S_{1}(X) S_{r-1}(X)\right\} \\
& \leq r 2^{r-1}\left\{\max _{i} b_{i} S_{r}(X)+\rho \gamma N^{-1} S_{0}(X) S_{r}(X)\right\},
\end{aligned}
$$

so that, since $S_{0}(X)=N$, we can take $k_{r 1}=r 2^{r-1}\left(\max _{i} b_{i}+\rho \gamma\right)$ and $k_{r 2}=$ $k_{r 4}=0$ in (2.6), and $r_{\max }^{(1)}=\infty$. Finally, for $(2.7), N V_{0 N}(X)=0$ and, for $r \geq 1$,

$$
\begin{aligned}
& N V_{r N}(X) \\
& \leq r^{2}\left\{2^{2(r-1)} \max _{i} b_{i} S_{2 r-1}(X)+\max _{i}\left(i^{-1} d_{i}\right) S_{2 r}(X)+\gamma(1-\rho) S_{2 r-1}(X)\right. \\
& \left.\quad+N^{-1} \rho \gamma\left(2^{2(r-1)} S_{1}(X) S_{2 r-2}(X)+S_{0}(X) S_{2 r-1}(X)\right)\right\}+\kappa S_{2 r}(X),
\end{aligned}
$$

so that we can take $p(r)=2 r$, and (assuming $i^{-1} d_{i}$ to be finite)

$$
k_{r 3}=\kappa+r^{2}\left\{2^{2(r-1)}\left(\max _{i} b_{i}+\rho \gamma\right)+\max _{i}\left(i^{-1} d_{i}\right)+\gamma\right\},
$$

and $k_{r 5}=0$ for any $r \geq 1$, and $r_{\max }^{(2)}=\infty$. In Assumptions 4.2, we can again take $r_{\mu}=1$ and $\zeta(k)=(k+1)^{7}$, giving $r(\zeta)=8, b(\zeta)=1$ and $\rho(\zeta, \mu)=16$.

\section{Acknowledgement}

ADB wishes to thank both the Institute for Mathematical Sciences of the National University of Singapore and the Mittag-Leffler Institute for providing a welcoming environment while part of this work was accomplished. MJL thanks the University of Zürich for their hospitality on a number of visits. 


\section{References}

[1] Arrigoni, F. (2003). Deterministic approximation of a stochastic metapopulation model. Adv. Appl. Prob. 35 691-720.

[2] Barbour, A. D. and Kafetzaki, M. (1993). A host-parasite model yielding heterogeneous parasite loads. J. Math. Biology 31 157-176.

[3] Barbour, A. D. and Luczak, M. J. (2008). Laws of large numbers for epidemic models with countably many types. Ann. Appl. Probab. 18 $2208-2238$.

[4] Eibeck, A. and Wagner, W. (2003). Stochastic interacting particle systems and non-linear kinetic equations. Ann. Appl. Probab. 13 845889.

[5] Kimmel, M. and Axelrod, D. E. (2002). Branching processes in biology. Springer, Berlin.

[6] Kretzschmar, M. (1993). Comparison of an infinite dimensional model for parasitic diseases with a related 2-dimensional system. J. Math. Analysis Applics 176 235-260.

[7] Kurtz, T. G. (1970). Solutions of ordinary differential equations as limits of pure jump Markov processes. J. Appl. Probab. 7 49-58.

[8] Kurtz, T. G. (1971). Limit theorems for sequences of jump Markov processes approximating ordinary differential processes. J. Appl. Probab. 8 $344-356$.

[9] LÉOnARD, C. (1990). Some epidemic systems are long range interacting particle systems. In: Stochastic Processes in Epidemic Theory, Eds J.-P. Gabriel, C. Lefèvre \& P. Picard, Lecture Notes in Biomathematics 86 170-183: Springer, New York.

[10] Luchsinger, C. J. (1999). Mathematical Models of a Parasitic Disease, Ph.D. thesis, University of Zürich.

[11] Luchsinger, C. J. (2001a). Stochastic models of a parasitic infection, exhibiting three basic reproduction ratios. J. Math. Biol. 42, 532-554. 
[12] Luchsinger, C. J. (2001b). Approximating the long term behaviour of a model for parasitic infection. J. Math. Biol. 42, 555-581.

[13] Pazy, A. (1983). Semigroups of Linear Operators and Applications to Partial Differential Equations, Springer, Berlin. 